\title{
Transient lift force on a blade during cutting of a vortex with non-zero axial flow
}

\author{
D. Curtis Saunders ${ }^{1}$ and Jeffrey S. Marshall ${ }^{1} \dagger$ \\ ${ }^{1}$ Department of Mechanical Engineering, The University of Vermont, Burlington, VT 05405, USA
}

(Received 27 October 2016; revised 21 March 2017; accepted 22 March 2017;

first published online 18 April 2017)

The problem of orthogonal penetration of a blade into the core of a vortex with non-zero axial flow was studied using a combination of scaling theory, a heuristic plug-flow model and full Navier-Stokes simulations. The particular focus of this paper was to understand the mechanics of the transient lift force that occurs during the initial penetration of the blade leading edge into the vortex core, and the relationship of this transient force to the steady-state lift force that develops due to the difference in vortex core radius over the blade surface. The three modelling approaches all lead to the conclusion that the maximum value of the lift coefficient for the transient blade penetration force is proportional to the impact parameter and inversely proportional to the axial flow parameter. This observation is used to develop a simple expression that collapses the predictions of the full Navier-Stokes simulations for lift coefficient over a wide range of parameter values.

Key words: aerodynamics, vortex flows, vortex interactions

\section{Introduction}

Unsteady forces on blades, airfoils and impellers due to chopping of vortex structures in the incident flow play an important role in many different applications. In helicopters that are either hovering or moving forward slowly, vortices shed from the main rotor are swept backward and impinge on the vehicle tail or are entrained into the tail rotor (Leverton, Pollard \& Wills 1977; Sheridan \& Smith 1980; Cary 1987). For fixed-wing aircraft, the effects of 'wake turbulence' due to interaction of an airplane with the wake vortices of a preceding airplane continues to be a leading cause of accidents, particularly among smaller aircraft. Wind turbines similarly ingest and chop vortices of the incident atmospheric flow (Churchfield et al. 2012; Shafii et al. 2013), particularly in rough topological conditions or when situated in a wind farm and subjected to the wake of upstream turbines. In many pumps, such as axial flow pumps, intake vortices and turbulent flow vortices in the intake flow are chopped by the pump impeller (Nagahara, Sato \& Okamura 2001). The streamwise hull vortices on torpedoes and submersibles, as well as vortices shed from upstream control surfaces, can be ingested into a propeller intake and chopped by the propeller blades (Felli, Camussi \& Guj 2009; Felli \& Falchi 2011). A similar phenomenon occurs in turbomachinery flows, where vortices shed from the upstream stator blades 
are carried downstream and are chopped by the rotor blades (Binder 1985), leading to turbulence generation.

Vortex cutting is a type of vortex reconnection process in which vortex lines within the vortex are cut and reconnect to vortex lines within the blade boundary layer. The problem of symmetric vortex cutting, with no ambient vortex axial flow, was examined experimentally by Weigand (1993) and using an inviscid computational approach by Marshall \& Grant (1996) for the problem of a vortex ring cut by a blade. Simulation of the full viscous problem of vortex cutting by a blade was reported by Liu \& Marshall (2004) for orthogonal interaction and by Filippone \& Afgan (2008) for cases with non-zero blade angle of attack. A recent detailed study of vortex reconnection during symmetric vortex cutting was presented by Saunders \& Marshall (2015), which presented full viscous simulations of vortex cutting and compared them to a simple analytical model that incorporates some aspects of the reconnection process.

A review of experimental and computational research on vortex cutting arising from orthogonal vortex-blade interaction problems is given by Coton et al. (2004). This review summarizes the results of a series of experimental studies of orthogonal vortex-blade interaction at high Reynolds number, which were performed during the late 1990s and early 2000s in the wind tunnel at Glasgow University (Doolan, Coton \& Galbraith 1999, 2001; Green, Doolan \& Cannon 2000; Early, Green \& Coton 2002; Wang et al. 2002; Green, Coton \& Early 2006). Visualization of vortex-body interaction was also conducted during this time period using low Reynolds number experiments in water, many of which made use of laser-induced fluorescence and particle image velocimetry for vortex imaging and measurement (Johnston and Sullivan 1992; Krishnamoorthy \& Marshall 1994, 1998; Marshall \& Krishnamoorthy 1997).

Cutting (or chopping) of a vortex with non-zero axial flow produces an impulsive lift force on the blade. Repeated vortex chopping produces vibrations, which can lead to material fatigue and system control problems. Vortex cutting is often responsible for low-frequency noise emitted by pumps and helicopters (Leverton et al. 1977; Paterson \& Amiet 1979; Ahmadi 1986; Howe 1989). Using the plug-flow models for vortex axial flow developed by Lundgren \& Ashurst (1989) and Marshall (1991), the response of the vortex core to instantaneous cutting by a thin blade was shown to respond in a manner analogous to the classic problem of a suddenly closed gate in a one-dimensional gas flow (Marshall 1994; Marshall \& Yalamanchili 1994). It is noted that the plug-flow model for axial motion on a vortex core results in a hyperbolic set of equations for vortex core radius and axial velocity that can be mapped into the one-dimensional gas flow equations, and consequently yields solutions analogous to the expansion fans and shock waves of compressible gas flow. Specifically, a "vortex expansion fan' is observed to propagate away from the blade on the downstream side of the vortex (relative to the vortex axial flow), over which the vortex core radius gradually increases from a reduced value near the blade surface to its ambient value far away from the blade. A compression wave that forms into a 'vortex shock' propagates away from the blade on the upstream side of the vortex, and the vortex core radius close to the blade surface on the upstream side increases relative to the ambient value. Experimentally, the vortex shock was observed to have the form of a travelling vortex breakdown which translates at the theoretically predicted vortex shock propagation speed (Krishnamoorthy \& Marshall 1994). The difference in vortex core radius on the upstream and downstream sides of the blade leads to a net pressure force in the direction of the ambient vortex axial flow. This steady-state vortex cutting force is imposed on the vortex as the vortex is cut and persists throughout the time 
period following vortex cutting (Marshall 1994). Full Euler equation simulations of the inviscid response of a vortex core with axial flow following instantaneous cutting were examined by Marshall \& Krishnamoorthy (1997) and Lee, Burggraf \& Conlisk (1998) for incompressible flow and by Yildirim \& Hillier (2013) for compressible flow, which show good agreement with the plug-flow model predictions for change in core radius and vortex wave speed.

Full viscous simulations of vortex cutting by a blade with non-zero vortex axial flow were reported by Liu \& Marshall (2004). The simulations demonstrated that a strong transient vortex cutting force is imposed on the blade during the time period in which the vortex is being cut by the blade leading edge, the magnitude of which decreases after the vortex is cut. The magnitude of this transient vortex cutting force was shown by Liu and Marshall to compare well to the experimentally observed lift force on the blade (Wang et al. 2002), even though the Reynolds number between simulation and experiment differed by three orders of magnitude. While vortex cutting requires the presence of finite fluid viscosity in order to occur, the observation that computational predictions and experimental measurements of the transient vortex cutting force agree well even when the Reynolds number is vastly different indicates that the transient vortex cutting force is of inviscid origin. In some cases examined, the magnitude of the transient vortex cutting force was found to be much larger than that of the steadystate vortex cutting force derived from the plug-flow vortex models with instantaneous vortex cutting. For such cases, the net lift on the blade might be observed to increase to a maximum value equal to the transient vortex cutting force as the leading edge of the blade penetrates into the vortex core, and then to decrease to the steady-state vortex cutting force value after the leading edge of the blade has fully penetrated through the vortex. On the other hand, if the transient vortex cutting force is smaller than the steady-state force, then the lift on the blade would increase monotonically throughout the vortex cutting process and eventually asymptote to the steady-state force value.

The objective of the current paper is to explain the underlying physics of the transient vortex cutting force through a combination of scaling analysis, heuristic modelling and full viscous flow simulations. Issues of particular interest include how the transient vortex cutting force depends on the various dimensionless parameters that govern the flow field and how the transient force compares to the steady-state vortex cutting force. A scaling analysis given in $\S 2$ is used to identify the source of the transient vortex cutting force, and results in a simple estimate for the magnitude of the peak transient vortex cutting force. Results from the method of characteristics analysis for the steady-state vortex cutting force are presented in $\S 3$, along with asymptotic approximations of the lift force for large and small values of the axial flow parameter. A heuristic model for the vortex response as the blade penetrates into the vortex core is developed in $\S 4$, which extends the vortex models of Lundgren \& Ashurst (1989) and Saffman (1990) to account for frictional force at the blade surface due to the vortex axial flow. The lift force on the blade predicted by the model exhibits different regimes, in one of which the transient vortex cutting force dominates and in the other the steady-state vortex cutting force dominates. The scaling estimate and heuristic model predictions are validated in $\S 5$ using full Navier-Stokes simulations for normal impact of a blade onto a vortex with finite axial flow. Conclusions are presented in $\S 6$.

\section{Scaling of the transient vortex cutting force}

In this section, a scaling analysis is developed to motivate an expression for how the transient vortex cutting force on the blade depends on the various dimensionless 
(a)

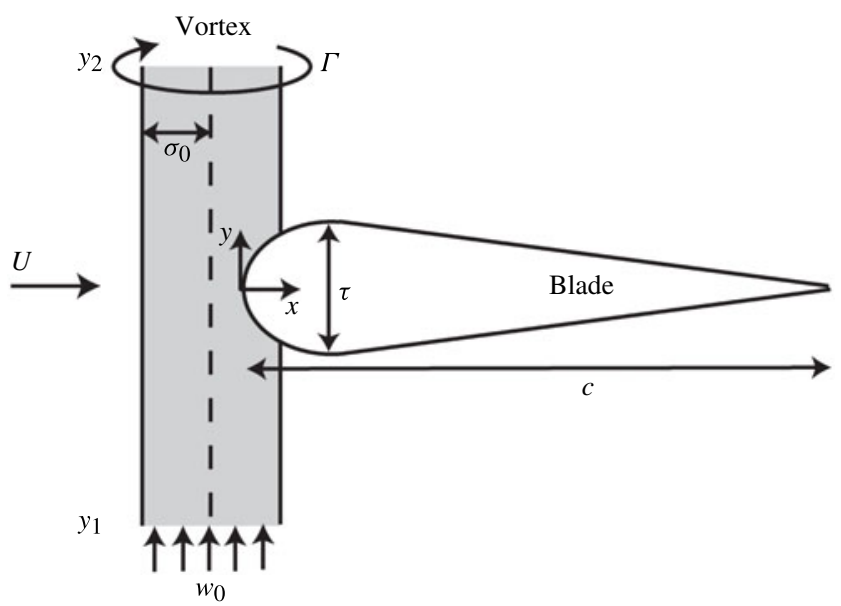

(b)

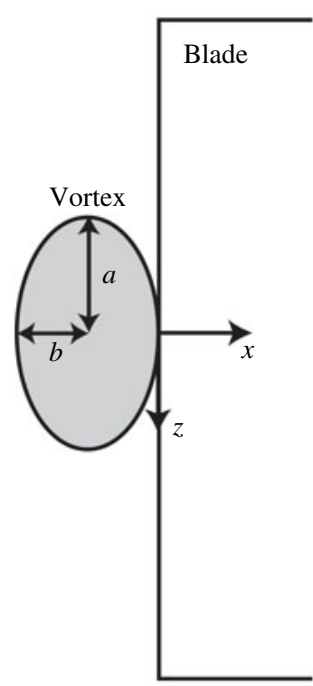

FIGURE 1. Schematic diagram showing the parameters used to describe the vortex cutting problem and the control volume (shaded) used in the scaling analysis, from both $(a)$ the side view and $(b)$ the top view.

parameters governing the flow field. The problem is described using a Cartesian coordinate system, oriented such that the uniform flow is in the $x$-direction, the normal vector of the blade centre plane is in the $y$-direction and the blade span is in the $z$-direction (figure 1). Orthogonal vortex-blade interaction is characterized by a number of parameters, including the blade chord $c$ and thickness $\tau$, the vortex circulation $\Gamma$, core radius $\sigma_{0}$ and maximum ambient axial velocity $w_{0}$, the blade speed relative to the vortex core $U$ and the fluid density $\rho$ and kinematic viscosity $\nu$. The vortex and blade Reynolds numbers are defined by $R e_{V} \equiv \Gamma / \nu$ and $R e_{B} \equiv U c / v$. The thickness parameter $T=\tau / \sigma_{0}$ is the ratio of the blade thickness to the vortex core radius. The impact parameter $I \equiv 2 \pi \sigma_{0} U / \Gamma$ is the ratio of the relative vortex-blade velocity to the maximum swirl velocity within the vortex. The axial flow parameter $A \equiv 2 \pi \sigma_{0} w_{0} / \Gamma$ is the ratio of the maximum vortex axial velocity to the maximum swirl velocity. Noting that the propagation speed of axisymmetric waves in the plug-flow vortex model is given by $c_{0}=\Gamma / \sqrt{8} \pi \sigma_{0}$, the axial flow parameter can be interpreted as analogous to a Mach number for the vortex flow.

A schematic diagram showing the different parameters used to describe vortex cutting is given in figure 1 . The region shaded grey in figure $1(a)$ is the vortex control volume $(\mathrm{CV})$, with lower and upper end points in the $y$-direction (along the vortex axis) at $y_{1}$ and $y_{2}$, respectively. Integrating the momentum equation over this control volume gives the lift force that the fluid exerts on the blade in the positive $y$-direction as

$$
L=-\frac{\mathrm{d}}{\mathrm{d} t} \int_{C V} \rho w \mathrm{~d} v
$$

where we assume that the ends of the $\mathrm{CV}$ on the vortex are sufficiently far from the blade that the axial velocity at each end is approximately equal, or $w\left(y_{2}, t\right)=w\left(y_{1}, t\right)$. The vortex cross-section is assumed to have approximately elliptical shape, with major 
semi-axis $a(\xi, t)$ and minor semi-axis $b(\xi, t)$, where $\xi$ denotes distance along the vortex axis. The effective radius $\sigma$ and the aspect ratio $\theta$ are defined by $\sigma \equiv(a b)^{1 / 2}$ and $\theta \equiv a / b=(\sigma / b)^{2}$. Assuming that the axial velocity is uniform across the vortex core (the plug-flow approximation), the lift force is given by

$$
L=-\pi \rho \frac{\mathrm{d}}{\mathrm{d} t} \int_{y_{1}}^{y_{2}} a b w \mathrm{~d} \xi .
$$

The transient vortex cutting force is caused both by the displacement of the vortex as the blade leading edge penetrates into the vortex core and by the vortex response on either side of the blade. The full Navier-Stokes simulations discussed in $\S 5$ indicate that the half-width of the vortex along the blade span, $a$, is nearly constant during the penetration process and that the maximum axial velocity $w_{\max }$ increases by approximately $50 \%$ and then decreases again as the blade penetrates into the vortex core. The largest change appears to be in the vortex radius $b$ in the direction of the uniform flow, which decreases from a value of approximately $\sigma_{0}$ prior to vortex cutting to nearly zero at the conclusion of cutting. For the purpose of forming a scaling estimate of the lift force, we assume that both $a$ and $w$ are constant in the integral in (2.2), equal to their initial values $\sigma_{0}$ and $w_{0}$, respectively, so that (2.2) becomes

$$
L \approx-\pi \rho \sigma_{0} w_{0} \int_{y_{1}}^{y_{2}} \frac{\mathrm{d} b}{\mathrm{~d} t} \mathrm{~d} \xi .
$$

It is noted that $\mathrm{d} b / \mathrm{d} t=-U / 2$ for all values of $\xi$ corresponding to positions on the blade that have penetrated into the vortex core, which has maximum extent $-\tau / 2<$ $\xi<\tau / 2$. However, the value of $b$ will also decrease on parts of the vortex on either side of the blade over a distance of scale $\sigma_{0}$. Based upon these approximations, (2.3) yields a scaling estimate for the maximum value of the transient vortex cutting force on the blade, $L_{t r, \max }$, as

$$
L_{t r, \text { max }} \cong \frac{\pi}{2} \rho U w_{0} \sigma_{0}\left(C_{1} \tau+C_{2} \sigma_{0}\right),
$$

where $C_{1}$ and $C_{2}$ are $O(1)$ constants. If we define a lift coefficient $C_{L}$ in terms of the ambient vortex axial velocity and core radius as $C_{L} \equiv L /\left(\rho w_{0}^{2} \sigma_{0}^{2} / 2\right)$, then the scaling estimate (2.4) can be written in terms of the axial flow parameter $A$, the impact parameter $I$ and the thickness coefficient $T$ as

$$
C_{L, t r, \max } \cong \frac{\pi I}{A}\left(C_{1} T+C_{2}\right) .
$$

\section{Steady-state vortex cutting force}

In addition to the transient force discussed in $\S 2$, there also exists a steady-state vortex cutting force that arises from the fact that the vortex core radius near the blade surface increases to a value $\sigma_{C}>\sigma_{0}$ on the upstream side of the core and decreases to a value $\sigma_{E}<\sigma_{0}$ on the downstream side of the core following vortex cutting. The difference in core radius leads to a net pressure force on the blade surface. A solution for this pressure force was obtained by Marshall (1994) using the method of characteristics to solve the one-dimensional plug-flow vortex model with instantaneous vortex cutting. For the configuration shown in figure $1(a)$ with $w_{0}>0$, 
the steady-state vortex cutting force $L_{s s}$ can be written in terms of the lift coefficient $C_{L}$ and the axial flow parameter $A$ as

$$
C_{L, s s}=\frac{2 \pi}{A^{2}} \ln \left(\frac{\sigma_{C}}{\sigma_{E}}\right) .
$$

The vortex core radii near the blade on the downstream (expansion) side and the upstream (compression) side, relative to the vortex axial flow, can similarly be written in terms of the axial flow parameter as

$$
\frac{\sigma_{E}}{\sigma_{0}}=\frac{1}{1+(\sqrt{2} / 2) A}
$$

and

$$
A^{2} \phi^{2}=\left(\phi^{2}-1\right) \ln (\phi)
$$

where $\phi \equiv \sigma_{C} / \sigma_{0}$ must be solved iteratively from (3.3).

In the limit of large axial flow parameters $(A \gg 1)$, (3.3) admits the asymptotic solution

$$
\phi \sim \exp \left(A^{2}\right)
$$

This solution requires that $\phi^{2} \gg 1$, and so has an error of only $1.8 \%$ at $A=2$. Substituting (3.4) and (3.2) into (3.1) gives an asymptotic solution for the steady-state vortex cutting force for $A \gg 1$ as

$$
C_{L, s s} \sim 2 \pi+\frac{2 \pi}{A^{2}} \ln \left(1+\frac{\sqrt{2}}{2} A\right) .
$$

For small axial flow parameters $(A \ll 1)$, (3.3) admits an asymptotic solution of the power series form

$$
\phi \sim 1+\frac{1}{\sqrt{2}} A+\frac{1}{2} A^{2}+O\left(A^{3}\right) .
$$

Using (3.6) and (3.2) in (3.1) and retaining two terms in the small parameter $A$ yields an asymptotic solution for the steady-state vortex cutting lift coefficient for $A \ll 1$ as

$$
C_{L, s s} \sim \frac{2 \sqrt{2} \pi}{A}+D A,
$$

where $D=5 \sqrt{2} \pi / 16 \cong 1.3884$ is an $O(1)$ constant.

\section{Heuristic model of vortex response to cutting}

This section develops a one-dimensional heuristic model of the vortex response during cutting and the development of the blade lift force using an approach motivated by the plug-flow vortex model of Lundgren \& Ashurst (1989) and the vortex tube reconnection model of Saffman (1990). The primary purpose of this model is to explore the relative role of the different forces acting on the vortex during cutting 
and the qualitative response of the vortex axial velocity and core radius and of the blade lift to these forces.

Accurate solution of (2.2) for the blade lift force during vortex cutting requires knowledge of how the vortex axial velocity and core radius change along the vortex as a function of time and position. As a blade penetrates into a vortex core, the vortex axial flow comes into contact with the blade surface resulting in a shear force that opposes the axial transport of fluid in the vortex. At the same time, the vortex comes into contact with the high pressure region near the blade leading edge, which generates an external pressure gradient along the vortex core. Finally, the blade penetration causes the vortex to become pinched, such that the semi-minor axis $b(\xi, t)$ reduces in time within the blade penetration region. These different effects cause changes in both the vortex core effective radius $\sigma(\xi, t)$ and the axial velocity $w(\xi, t)$. One approach to understanding the time and spatial variation of the core radius and axial velocity is the simplified one-dimensional model of Lundgren \& Ashurst (1989), which assumes the presence of a uniform axial velocity distribution (i.e. a 'plug flow') within the core. Application of a similar model to the vortex tube reconnection problem was given by Saffman (1990), in which the axial variation of vortex circulation caused by the vortex reconnection was shown to give rise to an internal pressure gradient within the vortex core. Saffman's model was found to be in reasonable qualitative agreement with data from full Navier-Stokes solutions of vortex tube reconnection (Shelley, Meiron \& Orszag 1993).

The equations of continuity and conservation of momentum for the plug-flow vortex model are given by

$$
\begin{gathered}
\frac{\partial}{\partial t}\left(\sigma^{2}\right)+\frac{\partial}{\partial \xi}\left(\sigma^{2} w\right)=0 \\
\frac{\sigma^{2}}{2}\left(\frac{\partial w}{\partial t}+w \frac{\partial w}{\partial \xi}\right)=-\frac{1}{\rho} \int_{0}^{\sigma} \frac{\partial p}{\partial \xi} r \mathrm{~d} r-\frac{a}{\rho} \tau_{w}
\end{gathered}
$$

where the pressure integral in (4.2) is across the vortex core, $\sigma \equiv(a b)^{1 / 2}$ is the equivalent core radius and the last term in (4.2) is added to account for the frictional force between the vortex axial flow and the blade leading edge. Following Saffman (1990), the pressure at a radius $r$ from the vortex axis can be expressed as a function of aspect ratio $\theta$ as

$$
\frac{p(r)-p_{\infty}}{\rho}=-\frac{\Gamma^{2} g(\theta)}{4 \pi^{2} \sigma^{4}}\left(2 \sigma^{2}-r^{2}\right)
$$

where $g(\theta)=\theta /\left(1+\theta^{2}\right)$ for $\theta>1$. Substituting (4.3) into (4.2) and performing the integration over $r$ gives

$$
\frac{\partial w}{\partial t}+w \frac{\partial w}{\partial \xi}=-\frac{\Gamma^{2} g(\theta)}{2 \pi^{2} \sigma^{3}} \frac{\partial \sigma}{\partial \xi}+\frac{3}{8 \pi^{2} \sigma^{2}} \frac{\partial}{\partial \xi}\left[\Gamma^{2} g(\theta)\right]-\frac{1}{\rho} \frac{\partial p_{\infty}}{\partial \xi}-\frac{2}{\rho b} \tau_{w} .
$$

Equation (4.4) reduces to the standard plug-flow model when $b=\sigma$ and $\Gamma$ is constant, with the addition of the external pressure gradient and shear force terms.

For simplicity, it is assumed that the vortex axis does not bend due to the inviscid interaction with the blade, so that we can set $\xi=y$. The external pressure gradient $\partial p_{\infty} / \partial y$, the shear stress $\tau_{w}$ and the variation in vortex circulation $\Gamma(y, t)$ can all be approximated using the results of full Navier-Stokes solutions for uniform flow past 
a blade (NACA0012 airfoil), which are given by Saunders \& Marshall (2015) for no axial flow and in $\S 5$ of the current paper with axial flow. For the external pressure variation, results from Saunders \& Marshall (2015) yield

$$
p_{\infty} \cong 0.16 \rho U^{2} \exp \left[-(y / \tau)^{2}\right],
$$

so that the external pressure gradient force per unit mass $F_{P}$ acting on the vortex is

$$
F_{P}=-\frac{\partial\left(p_{\infty} / \rho\right)}{\partial y} \cong 0.32 U^{2}\left(y / \tau^{2}\right) \exp \left[-(y / \tau)^{2}\right] .
$$

The effect of this external pressure gradient is to produce an axial flow moving away from the vortex cutting region, thus thinning the vortex core near the location of vortex cutting and increasing the vortex core radius on both sides of the blade.

The blade shear stress $\tau_{w}$ plays a critical role in reducing the axial velocity within the vortex core in the vortex cutting region. The shear stress is approximated by

$$
\tau_{w} \cong \mu w f(t) / \delta,
$$

where $\delta$ is a characteristic boundary layer thickness and $f(t)$ is the spanwise length of the region on the blade surface over which the shear stress acts. The function $f(t)$ can be regarded as a type of ramp function for the shear stress, which varies from zero when the vortex core axial flow first makes contact with the blade leading edge to approximately unity once the blade has penetrated half-way through the vortex core. For convenience, the current study assigned $f(t)$ to be a simple hyperbolic tangent function given by $f(t)=\tanh \left(2 t / t_{\text {cut }}\right)$. Here, $t=0$ corresponds to the time at which the blade leading edge first contacts the vortex and $t_{c u t}=2 \sigma_{0} / U$ is the nominal cutting time. The length scale $\delta$ is assumed to be proportional to the $99 \%$ stagnation-point boundary layer thickness $\delta_{s p}$ at the blade leading edge, given by

$$
\delta_{s p}=2.40(\nu / s)^{1 / 2} \cong(v \tau / U)^{1 / 2},
$$

using the estimate $s \cong 5.3 U / \tau$ for strain rate $s$ at the blade leading edge (Saunders $\&$ Marshall 2015). The shear stress from (4.7) becomes $\tau_{w} \cong C_{\text {shear }} \mu w f(t) / \delta_{s p}$, where $C_{\text {shear }}$ is a constant of proportionality, so that using (4.8) the shear force per unit mass acting on the vortex (i.e. the last term in (4.4)) can be written as

$$
F_{S}=-\frac{2 \tau_{w}}{\rho b} \cong-2 C_{\text {shear }} \frac{w}{b}\left(\frac{\mu U}{\rho \tau}\right)^{1 / 2} f(t) .
$$

The shear stress on the blade surface was measured for a range of different parameter values in the full Navier-Stokes simulations described in $\S 5$. In each case, (4.9) was used to estimate the coefficient $C_{\text {shear }}$ from the computational data. The values obtained varied between 4 and 10 . While this is a fairly wide range of variation, no doubt a consequence of the fact that the above equation for wall shear stress is just a rough estimate, the computed lift force on the blade was fortunately found not to be particularly sensitive to this coefficient (as seen from comparison of predictions with both $C_{\text {shear }}=4$ and 10 ).

The 'circulation gradient force' $F_{C}$ is given by the second term on the right-hand side of (4.4), or

$$
F_{C}=\frac{3}{8 \pi^{2} \sigma^{2}} \frac{\partial}{\partial y}\left[\Gamma^{2} g(\theta)\right]
$$



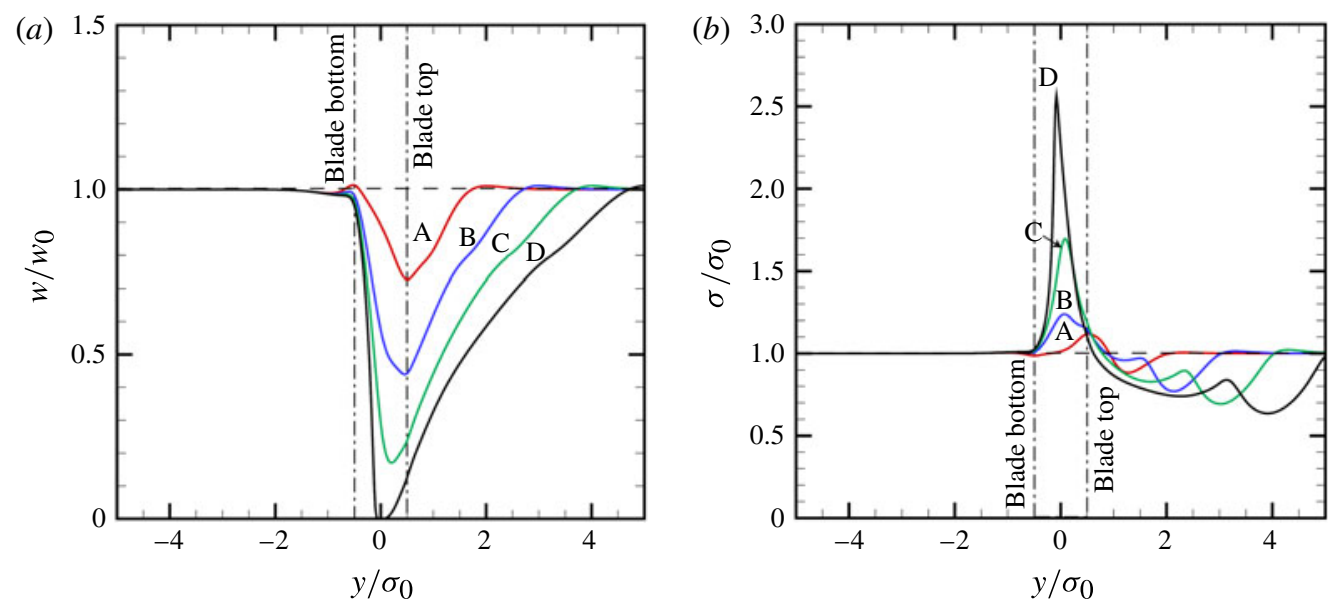

FIGURE 2. Computational results for $(a)$ vortex axial velocity and $(b)$ effective core radius from the plug-flow model for a case with dimensionless parameter values $I=1, A=2$, $T=1$ and $R e_{A}=500$. The plots are drawn for times $w_{0} t / \sigma_{0}=0$ (dashed), 1 (A, red), 2 (B, blue), $3(\mathrm{C}$, green) and 4 ( $\mathrm{D}$, black), where the last time coincides with the vortex cutting time.

This force is induced by the internal pressure gradient associated with the axial gradient in vortex circulation that arises as a consequence of vortex reconnection, and it featured prominently in the vortex tube reconnection model of Saffman (1990). The aspect ratio can be computed in terms of the minor axis length $b$ and the effective radius $\sigma$ using $\theta=(\sigma / b)^{2}$, where a smoothly varying approximation for $b$ is assumed in the form

$$
b(y, t) \cong 1-(U t / 2) \exp \left[-(2 y / \tau)^{2}\right] .
$$

The vortex circulation is approximated using data from the full Navier-Stokes simulation (Saunders \& Marshall 2015) as

$$
\Gamma(y, t) \cong \Gamma_{0}\left[1-\tanh \left(t / t_{c u t}\right)\right] \exp \left[-(2 y / \tau)^{2}\right] .
$$

Computations were performed using the McCormack finite-difference algorithm to solve (4.1) and (4.4) with time step $w_{0} \mathrm{~d} t / \sigma_{0}=0.001$ and spatial step size $\mathrm{d} x / \sigma_{0}=0.02$. Computations were performed with values of the shear coefficient $C_{\text {shear }}$ of 4 and 10 . Example solutions for effective core radius and axial velocity at four different times during the vortex cutting process are plotted in figure 2 for a case with dimensionless parameter values $I=1, A=2, T=1, R e_{A}=500$ and $C_{\text {shear }}=10$. A set of plots for this computation showing the dimensionless shear force $F_{S} / \sigma_{0} w_{0}^{2}$, the dimensionless external pressure gradient force $F_{P} / \sigma_{0} w_{0}^{2}$ and the dimensionless 'circulation gradient' force $F_{C} / \sigma_{0} w_{0}^{2}$ is given for the same four times in figure 3. Additional computations were performed with different combinations of these forces turned off in order to understand the role that each force plays in the overall solution.

The vortex flow field in figure 2 is divided into an upstream 'compression region' $(y<0)$ and a downstream 'expansion region' $(y>0)$ in which the core is axially compressed or expanded by the axial flow, respectively, and 'upstream' and 'downstream' are with respect to the core axial velocity. The vortex axial velocity 

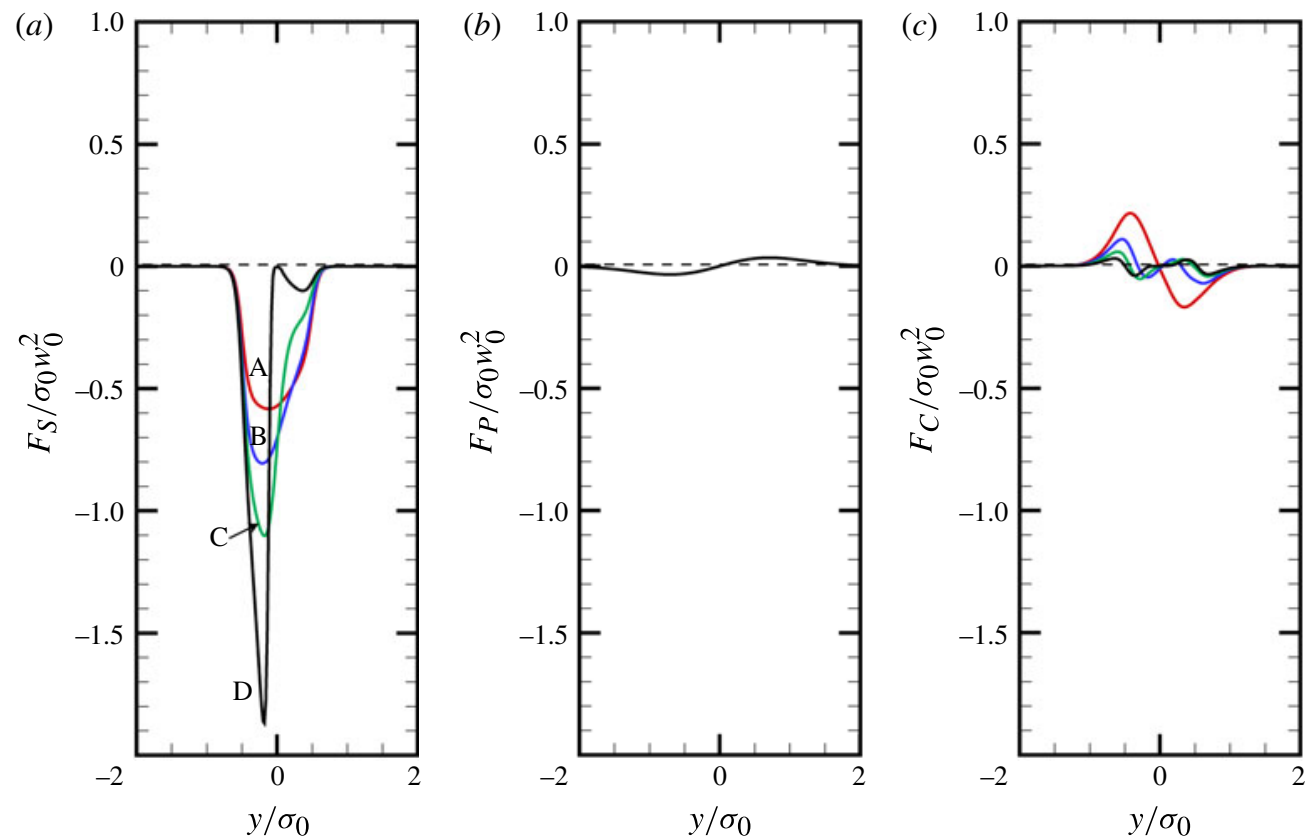

FIGURE 3. Plots showing variation along the vortex core of $(a)$ the shear force $F_{S},(b)$ the pressure gradient force $F_{P}$ and $(c)$ the vortex circulation gradient force $F_{C}$, per unit mass, at times $w_{0} t / \sigma_{0}=0$ (dashed), 1 (A, red), 2 (B, blue), $3(\mathrm{C}$, green) and 4 (D, black), for the same case as shown in figure 2. The pressure gradient force does not change in time.

is decreased near the blade and within a region lying between the blade surface and the vortex expansion far downstream of the blade due to the strong shear force between the blade leading edge and the vortex axial flow. The shear force similarly results in an increase in vortex effective core radius $\sigma$ immediately upstream of the blade leading edge and a corresponding decrease in $\sigma$ downstream of the blade. The increase (decrease) in effective vortex core radius on the upstream (downstream) side of the vortex from the cutting blade is consistent with the experimental observations and inviscid computations of Marshall \& Krishnamoorthy (1997) and the full Navier-Stokes simulations of Liu \& Marshall (2004). From figure 3, we observe that both the external pressure gradient force and the circulation gradient force have much smaller magnitudes than the shear force, so the vortex core response to the blade is governed primarily by the shear force.

The blade lift coefficient $C_{L}$ is obtained by computing the lift force by numerical evaluation of the integral in (2.2) using the trapezoidal rule with the computed values of core radius and axial velocity from solution of (4.1) and (4.4). The lift coefficient exhibits a peak value $C_{L, \max }$ shortly before the end of the vortex cutting process and then decreases at later times. A series of computations was conducted to determine how $C_{L, \max }$ varies with the values of the dimensionless parameters $A, I$ and $T$ for both $C_{\text {shear }}=4$ and 10 , the results of which are plotted in figure 4 . The vortex model solutions are compared to the scaling estimate (2.5) for the maximum transient vortex cutting force, which is indicated in these figures by line A. In comparing to the scaling solution for the transient lift force, we select the coefficients $C_{1}$ and 


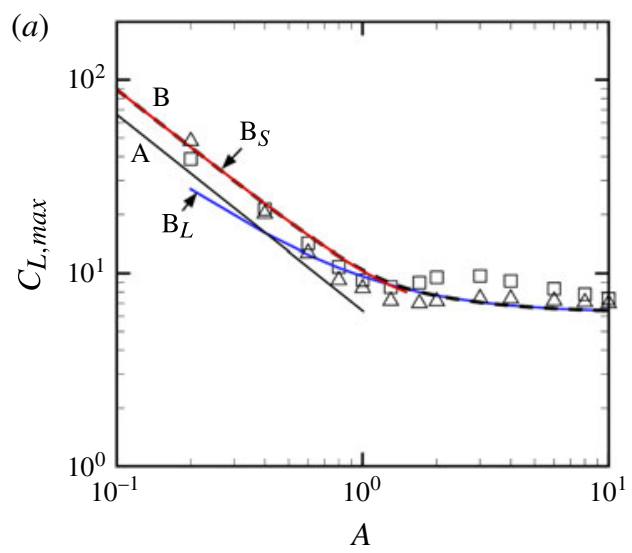

(b)
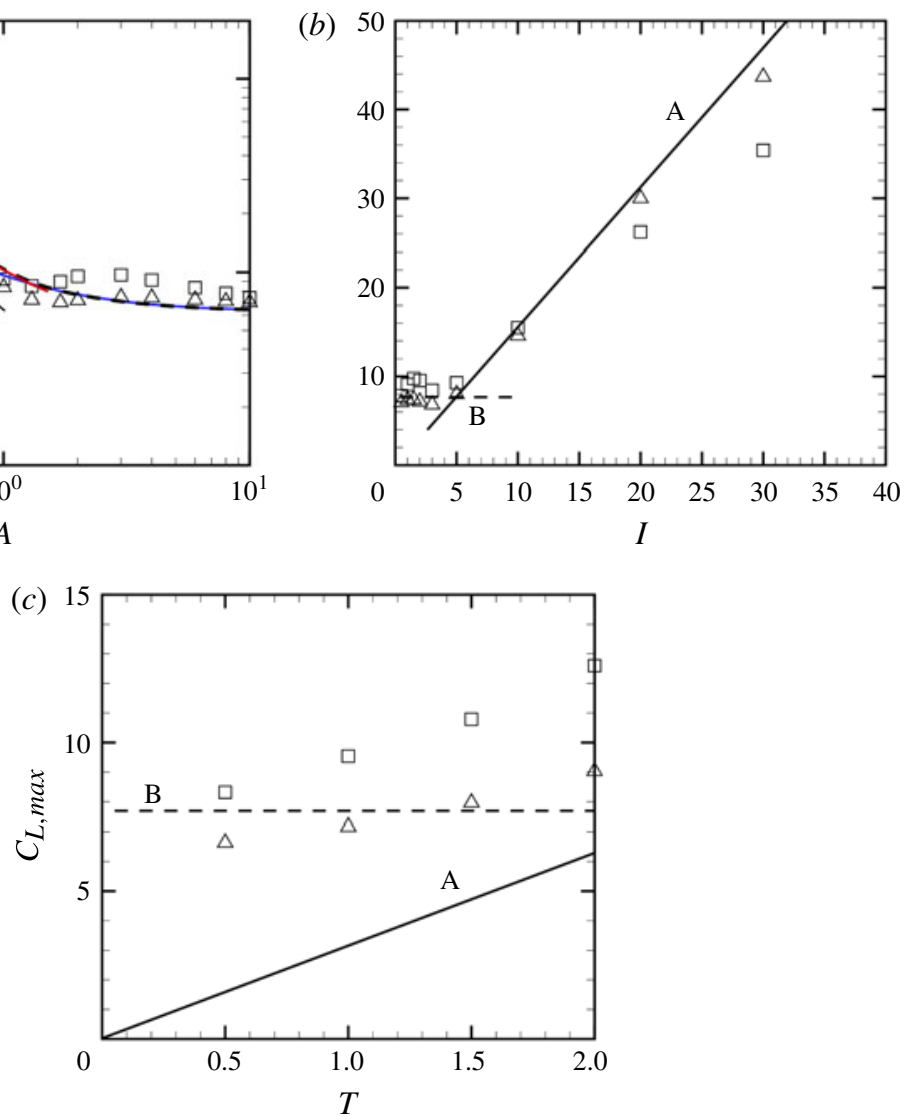

FIGURE 4. Variation of lift coefficient predicted from the plug-flow vortex model with (a) axial flow parameter (with $I=2$ and $T=1$ ), $(b)$ impact parameter (with $A=2$ and $T=1$ ) and $(c)$ thickness parameter (with $A=2$ and $I=2$ ). The plug-flow model results are for $C_{\text {shear }}=4$ (squares) and 10 (triangles). Line A (solid black) represents the scaling estimate (2.5) for the transient vortex cutting force with $C_{1}=1$ and $C_{2}=0$ and line B (dashed) is the steady-state vortex cutting force prediction obtained by solving (3.1)-(3.3). The asymptotic solutions for the steady-state vortex cutting force are indicated in $(a)$ by line $\mathrm{B}_{L}$ (blue) for large axial flow parameters and by line $\mathrm{B}_{S}$ (red) for small axial flow parameters.

$C_{2}$ in (2.5) as $C_{1}=1$ and $C_{2}=0$ in figure 4 , which appear to give the best fit to the data. The steady-state vortex cutting force was obtained by iterative solution of (3.3) for $\phi=\sigma_{C} / \sigma_{0}$ using the false position method and plugging the result into (3.1), with the result indicated by line B in figure 4. In figure 4(a), the value of $C_{L, \max }$ is plotted versus the axial flow parameter $A$ using a $\log -\log$ plot, where $A$ varies over the interval $(0.1,10)$. In this plot, we also show the solutions obtained from the asymptotic expansions (3.5) and (3.7) for the steady-state vortex cutting force at large and small values of $A$, indicated in figure $4(a)$ by lines $\mathrm{B}_{L}$ and $\mathrm{B}_{S}$, respectively. The computational solutions from the plug-flow model are in good agreement with the steady-state vortex cutting force solution for all values of $A$, as well as in reasonably good agreement with the transient vortex cutting force solution (2.5) for $A<1$. We also note that the two asymptotic solutions (3.5) and (3.7) for 
the steady-state vortex cutting force are close to the full solution for $A<1.2$ and for $A \geqslant 1.2$, respectively. In the figure $4(b)$, showing variation of $C_{L, \max }$ with the impact parameter $I$, the transient vortex cutting force prediction (2.5) agrees well with the computational solution for $I>5$, and the steady-state vortex cutting solution agrees with the computational data in the range $I<5$, for which the transient force prediction is less than the steady-state force prediction. Figure $4(c)$ shows the variation of $C_{L, \max }$ with the thickness parameter $T$. For low values of $T$ the plug-flow model predictions for $C_{L, \max }$ are not very sensitive to the value of $T$, and are given with reasonable accuracy by the steady-state solution. For large values of $T$ (e.g. $T>4$ ) the plug-flow model predictions agree well with the transient lift force model (2.5); however, results with $T$ larger than approximately 2 are not physically meaningful, since for such cases the vortex bends significantly prior to impingement on the vortex (Marshall \& Yalamanchili 1994), and they are therefore not shown in figure 4.

\section{Full Navier-Stokes simulations}

The previous sections discuss some of the physics that gives rise to the transient and steady-state vortex cutting forces on the blade in terms of both analytical solutions and a one-dimensional heuristic model based on the plug-flow approximation. This section seeks to examine the validity of these model predictions using full NavierStokes simulations of a blade with zero angle of attack cutting through the core of a vortex with non-zero axial flow.

\subsection{Numerical method}

The Navier-Stokes equations were solved in primitive-variable form using a finite-volume method (Lai 2000) on a block-structured mesh with hexahedral elements and eight implicitly coupled blocks. The grid was designed to provide smooth grid cell variation and high spatial accuracy in the region surrounding the blade leading edge, where the vortex cutting occurs (figure $5 a$ ). The computational algorithm stores dependent variable values at the cell centres and calculates second-order accurate approximations of the diffusive and convective fluxes at the cell boundaries. The momentum and continuity equations are coupled using the pressure implicit with splitting of operator (PISO) algorithm (Issa 1985). Additional numerical stability was achieved by weighting the time derivative between a second-order derivative approximation and a first-order upwind with a 90-10 ratio.

The computations were initialized with a columnar vortex with strength $\Gamma$ convected by a uniform flow with speed $U$ toward a fixed blade. The variation of swirl velocity and axial velocity with radius within the initial columnar vortex were specified as Gaussian functions. The initial position of the vortex axis was located approximately four vortex core radii upstream of the blade leading edge in order to allow the blade boundary layer time to grow in a natural way as the vortex approaches. When initialized as a Gaussian vortex, the vortex swirl $\left(u_{\theta}\right)$ and axial $(w)$ velocity fields were observed to remain Gaussian by the time the vortex reached the blade leading edge, with form

$$
u_{\theta}=\frac{\Gamma}{2 \pi r}\left[1-\exp \left(-r^{2} / \sigma_{0}^{2}\right)\right], \quad w=w_{0} \exp \left(-r^{2} / \sigma_{0}^{2}\right)
$$

The vortex maximum axial velocity and core radius at a time just before onset of cutting of the vortex by the blade were measured, and are denoted by $w_{0}$ and $\sigma_{0}$, respectively. 

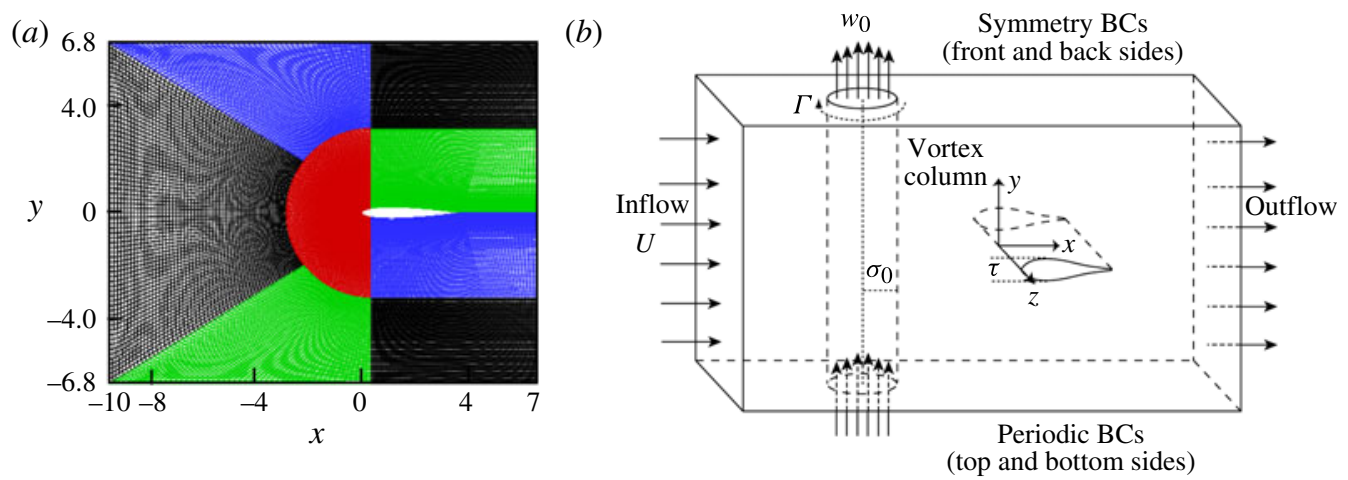

FIgURE 5. (a) Cross-sectional view of the computational grid in the plane $z=0$ and (b) schematic diagram showing boundary conditions, as used for the full Navier-Stokes simulations. The inlet and outlet planes are at $x=-10$ and $x=7$, respectively, and the blade span length is equal to 10 .

Standard inflow and outflow boundary conditions were used for the boundary planes in the $x$-direction, and periodic and symmetry boundary conditions were used in the $y$ - and $z$-directions, respectively, as shown in figure $5(b)$. The blade was a NACA symmetric airfoil with chord length $c$, thickness $\tau$ and with the leading edge lying on the line $x=0$. The computational domain spanned the region $-10 \leqslant x / \sigma_{o} \leqslant 7$, $-6.8 \leqslant y / \sigma_{o} \leqslant 6.8$ and $-5 \leqslant z / \sigma_{o} \leqslant 5$, where the blade centre plane is given by the intersection of the blade with the $y=0$ plane. The vortex axis was initialized on the central plane $\left(z_{0}=0\right)$ at a location $x_{0} / \sigma_{o}=-4.4$ upstream of the blade leading edge, and the inlet plane was located upstream of the blade leading edge at $x_{\text {inlet }} / \sigma_{o}=-10$.

The values of the various dimensionless parameters used for the Navier-Stokes computations are listed in table 1. The vortex Reynolds number is set at $\operatorname{Re}_{V}=1500$ for all cases examined. The impact parameter $I$ varies from 1.4 to 44.5 , the axial flow parameter $A$ varies from 0.06 to 11 and the thickness parameter $T$ varies from 0.13 to 1.05 . The impact parameter is maintained at a sufficiently large value that no boundary layer separation is observed prior to impact of the blade onto the vortex. The blade Reynolds number $R e_{B}$ varies inversely to the impact parameter, ranging from 2000 to 15000 for the different cases examined. A dimensionless time step of $\Delta t=0.06$ was chosen to ensure a Courant-Friedrichs-Lewy (CFL) number of less than unity for all computations.

All length variables are non-dimensionalized using the ambient vortex core radius $\sigma_{0}$, velocity variables are non-dimensionalized by the free stream velocity $U$, time is non-dimensionalized by $\sigma_{0} / U$ and vorticity is non-dimensionalized by the inverse time scale $U / \sigma_{0}$. Pressure and shear stress are non-dimensionalized by $\rho U^{2}$, where $\rho$ is the fluid density.

Grid independence of the simulation results was examined by repeating the run for Case 5 with four different meshes, where the total number of grid points varied by a factor of 4.4 between the coarsest and finest meshes. The meshes are labelled as mesh A-D, with the number of grid points varying from 860886 to 3750288 points. Grid independence was evaluated by calculating the maximum lift coefficient on the blade during vortex cutting, where all computations and grids had the same parameter values, domain size and block mesh structure. The results are shown in table 2, including the number of grid points and the maximum value of the lift coefficient for each mesh. 


$\begin{array}{cccccc}\text { Case } & T=\tau / \sigma_{0} & R e_{B} & I=\frac{2 \pi \sigma_{o} U}{\Gamma} & A=\frac{2 \pi \sigma_{o} w_{0}}{\Gamma} & \text { Symbol (figure 15) } \\ 1 & 0.48 & 15000 & 11.6 & 0.06 & + \\ 2 & 0.48 & 15000 & 11.6 & 0.3 & \triangle \\ 3 & 0.48 & 15000 & 11.6 & 0.6 & * \\ 4 & 0.48 & 15000 & 11.6 & 3.0 & \square \\ 5 & 0.48 & 15000 & 11.6 & 6.0 & \bullet \\ 6 & 0.48 & 15000 & 11.6 & 11.0 & \bullet \\ 7 & 0.48 & 2000 & 1.4 & 0.6 & \text { NA } \\ 8 & 0.48 & 7500 & 5.7 & 0.6 & \times \\ 9 & 0.48 & 38500 & 27.0 & 0.6 & \mathbf{\square} \\ 10 & 0.48 & 57500 & 44.5 & 0.6 & \text { \# } \\ 11 & 0.13 & 15000 & 11.6 & 0.6 & \diamond \\ 12 & 0.26 & 15000 & 11.6 & 0.6 & \nabla \\ 13 & 1.05 & 15000 & 11.6 & 0.6 & \end{array}$

TABLE 1. Values of the dimensionless parameters for the reported computations.

Mesh Number of grid points $C_{L, \max } \%$ difference with previous grid

$\begin{array}{cccc}\text { A } & 860866 & 12.6 & \\ \text { B } & 1459808 & 12.3 & 2.4 \\ \text { C } & 2446848 & 13.6 & 9.5 \\ \text { D } & 3750288 & 13.8 & 1.4\end{array}$

TABLE 2. Results of grid independence study, comparing results for meshes (A-D).

The maximum lift coefficient had a difference of $1.4 \%$ between the two finest meshes, and all subsequent computations were performed with the finest mesh $\mathrm{D}$.

The effect of domain size was also considered to ensure the accuracy of the numerical simulations. The most important parameter was the distance between the initial position of the vortex core and the inlet plane. If this distance was set too small, the induced velocity from the vortex caused weak positive $y$-vorticity to be generated on the inlet plane, which propagates towards the blade behind the vortex. When this vorticity reaches the blade leading edge it diffusively cancels with the uncut portion of the vortex core that was stretched around the blade leading edge. Several domains were examined, and the mesh in figure 5(a) was chosen in order to ensure that the strength of this inlet vorticity was small and that it did not reach the blade until very late in the computation.

\subsection{Vortex cutting with axial flow}

The vortex cutting process occurs due to the diffusive cross-cancellation of vorticity within the vortex core to that on the blade leading edge. To illustrate this process, the positive and negative vortex strength measures, defined by

$$
\Gamma^{+}=\int_{L} \omega_{y}^{+} \mathrm{d} x, \quad \Gamma^{-}=\int_{L} \omega_{y}^{-} \mathrm{d} x,
$$


(a)

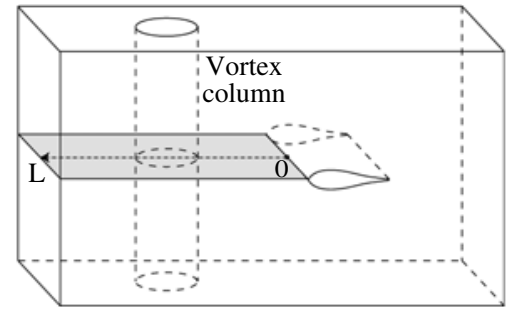

(b)
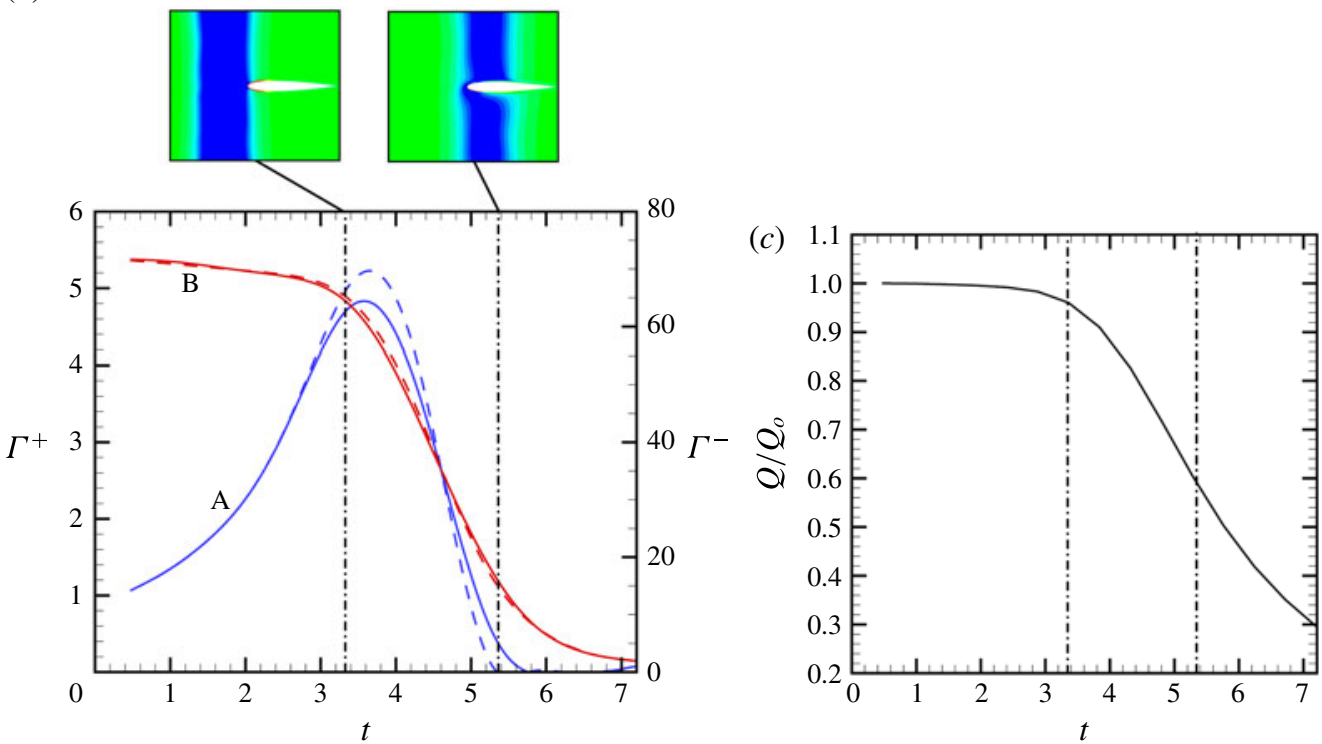

FIgURE 6. (a) The line, L, and plane (shaded region) over which the vortex strength, $\Gamma$, and flow rate, $Q$, were calculated in $(b)$ and $(c)$, respectively. (b) Positive and negative vortex strength measures, $\Gamma^{+}$(A, blue curve) and $\Gamma^{-}$(B, red curve), versus dimensionless time for Case 5 (solid lines) and for Case 5 with no axial flow (dashed lines). The circulation was calculated along a line extending out from the blade front in the $-x$ direction as shown in $(a)$. (c) Flow rate, $Q$, in the $y$-direction along the blade symmetry plane $(y=0)$, non-dimensionalized by the initial value. Vertical dashed-dotted lines correspond to the nominal starting and ending times for vortex cutting $(t=3.36$ and 5.36, respectively), as shown in the insert to $(b)$.

where

$$
\omega_{y}^{+}=\left\{\begin{array}{ll}
\omega_{y} & \text { if } \omega_{y}>0 \\
0 & \text { if } \omega_{y} \leqslant 0
\end{array} \quad \text { and } \quad \omega_{y}^{-}= \begin{cases}0 & \text { if } \omega_{y}>0 \\
-\omega_{y} & \text { if } \omega_{y} \leqslant 0\end{cases}\right.
$$

were calculated along a line $\mathrm{L}$ on the blade symmetry plane as shown in figure $6(a)$. We recall that the $y$-coordinate direction coincides with the direction along the axis of the ambient vortex. The vortex initialized in this study possessed negative $y$-vorticity within the vortex core. As the vortex core approached the blade, the induced velocity created a boundary layer of positive $y$-vorticity along the blade leading edge, the growth of which corresponds to the increase in positive circulation in figure $6(b)$. As the blade began to penetrate into the vortex core $(t=3.4)$, the induced positive $y$ vorticity in the blade boundary layer diffusively cancelled with the negative $y$-vorticity 
in the vortex core. This vorticity diffusion caused the vortex lines within the core to be cut and to reconnect to the vortex lines within the blade boundary layer, decreasing both the positive and negative circulation measures. Once the vortex centreline passes over the blade leading edge, this induced vorticity in the leading edge boundary layer changes sign, which in turn stops the vortex cutting process as the positive circulation measure approaches zero (figure $6 b$ ). As time advances further, the uncut portion of the vortex stretches over the blade leading edge to form a vortex sheet, but it cannot be cut due to a lack of positive $y$-vorticity in the boundary layer. As the vortex advects sufficiently far downstream, the induced velocity from the stretched, uncut vortex sheet increases in strength relative to that from the main vortex and positive $y$-vorticity will again form in the blade leading edge boundary layer, indicating a second, slower stage of vortex cutting.

A comparison of the time variation of $\Gamma^{+}$and $\Gamma^{-}$is also given in figure $6(b)$ between a computation with ambient axial vortex flow (Case 5) and the same run but with no ambient axial vortex flow. The results for $\Gamma^{-}$(corresponding to the vorticity within the vortex structure) for the computations with and without ambient axial flow are nearly identical. The positive vortex strength measure $\Gamma^{+}$(corresponding to the vorticity in the blade boundary layer) is slightly higher for the computation without ambient axial flow near the start of the blade penetration into the core and slightly lower the end of the vortex cutting process compared to the case with axial flow, but the differences are small. Overall, we conclude that axial core flow does not have a large influence on the vortex cutting process.

The volumetric flow rate in the axial direction through the plane extending out from the centre of the blade is plotted in figure $6(c)$ as a function of time. The flow rate depends on both the magnitude of axial velocity within the core and the core radius of the vortex. The flow rate remains relatively constant until the cutting process begins. Once vortex cutting starts, the axial flow rate decreases sharply. It then continues to decrease more gradually as the core remnant is stretched along the blade leading edge. A time series of contour plots of both the axial velocity and $y$-component of vorticity in the $x-z$ plane are given in figure 7 . After the cutting process ends following the change of sign of the leading edge boundary layer vorticity, the remaining core deforms as it is stretched along the span of the blade (figure $7 c, f$ ).

While the change in sign of induced $y$-vorticity nominally corresponds to when the centre of the vortex core passes over the blade leading edge, both the axial flow and the blade thickness parameter, $T$, were found to affect this change in sign. Figure 8 shows a time series of contour plots in the $x-y$ plane for Cases 5 and 13. In Case 5 , the blade thickness parameter is set to $T=0.48$ and the vorticity along the blade leading edge begins to change sign as the core centre passes over the blade leading edge. However, the presence of upward axial flow causes this change in sign to occur first on the top surface of the blade (figure $8 c, f)$. In figure $8(d-f)$, the blade thickness is increased such that $T=1.05$. In this case, as the vortex core passes over the blade leading edge, the induced $y$-vorticity does not change sign but instead remains positive within a region directly underneath the stretched vortex sheet (figure $8 c, f$ ). Vortex lines for the case with $T=1.05$ (Case 13) are shown in figure 9 from two different perspectives. The axial velocity within the vortex core causes the vortex lines to swirl around the core as they travel upwards. Figure $9(b)$ shows a contour plot of $y$-vorticity on the blade surface projected onto the $y-z$ surface, viewed from a perspective looking in the $x$-coordinate direction. The change in sign of $y$-vorticity occurs along the ends of the blade span after the vortex centre passes by the blade leading edge, but in the blade centre the change in sign is opposed by the velocity induced by the stretched 


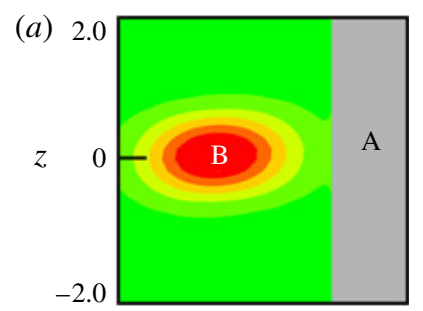

(b)

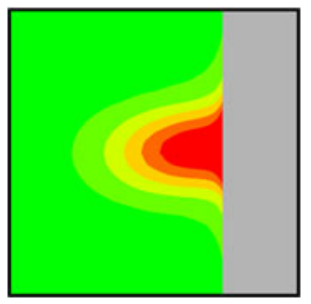

(e)

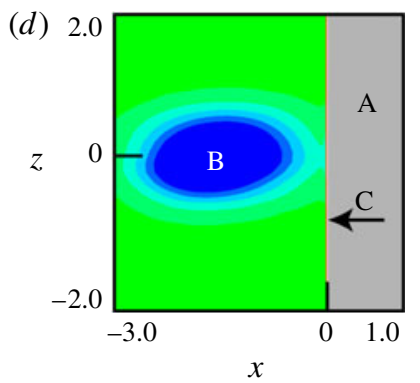

(c)

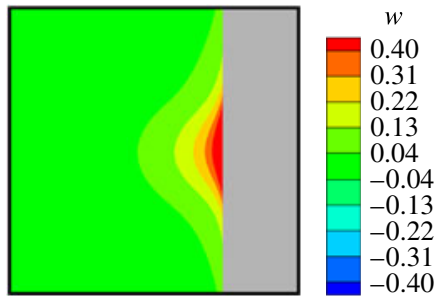

$(f)$

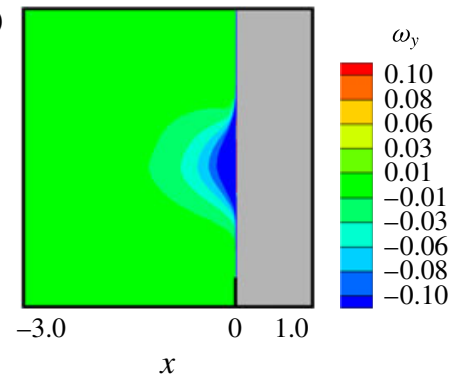

FIgURE 7. Time series from Case 5 showing contours of axial velocity $(a-c)$ and axial vorticity $(d-f)$ in the blade symmetry plane $(y=0)$ at times $(a, d) t=2.88,(b, e) 4.08$, and $(c, f)$ 5.28. The blade surface is labelled as A, vortex core as B and blade boundary layer as $\mathrm{C}$.
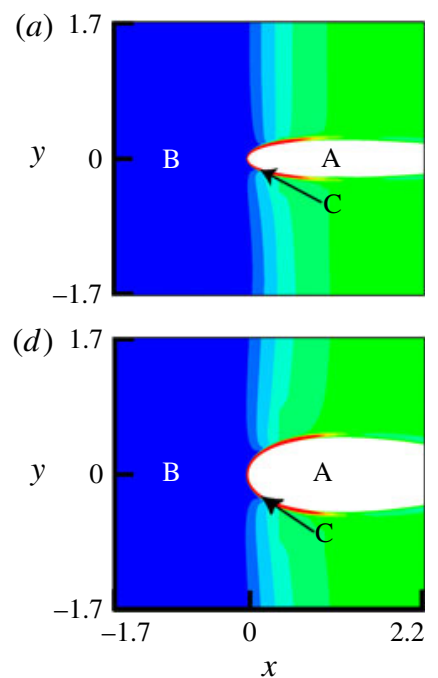

(b)

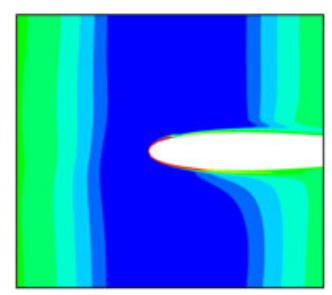

(e)

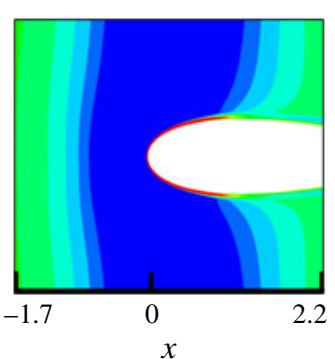

(c)

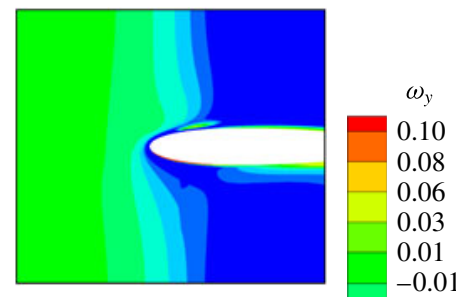

$(f)$

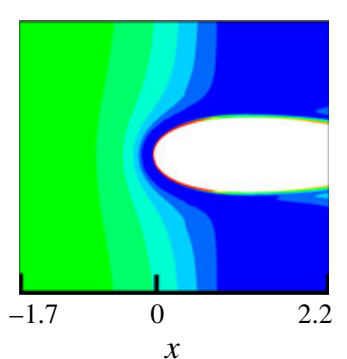

$-0.03$

$-0.06$

$-0.08$

$-0.10$

FIGURE 8. Contours of the vorticity component $\omega_{y}$ in a plane $z=0$ along blade leading edge for cases with thickness parameter values $T=0.48$ (Case 5, $a-c$ ) and $T=1.05$ (Case $13, d-f)$ at times $(a, d) t=3.6,(b, e) 4.8$ and $(c, f) 6$. The blade cross-section is labelled as $\mathrm{A}$, vortex core as $\mathrm{B}$ and blade boundary layer as $\mathrm{C}$.

(uncut) portion of the vortex that wraps around the blade leading edge. Also shown are the vortex lines that have been cut from the vortex core and have reconnected with the vortex lines from within the blade boundary layer. 
(a)

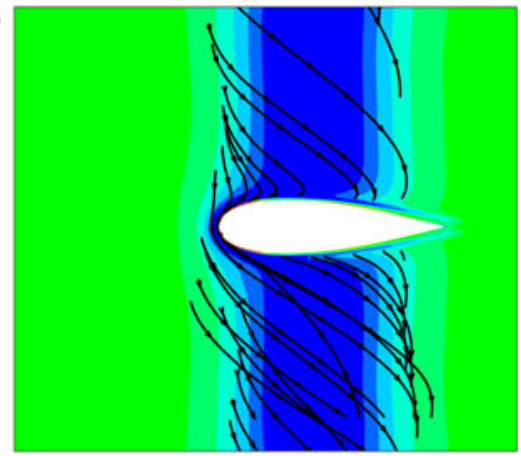

(b)

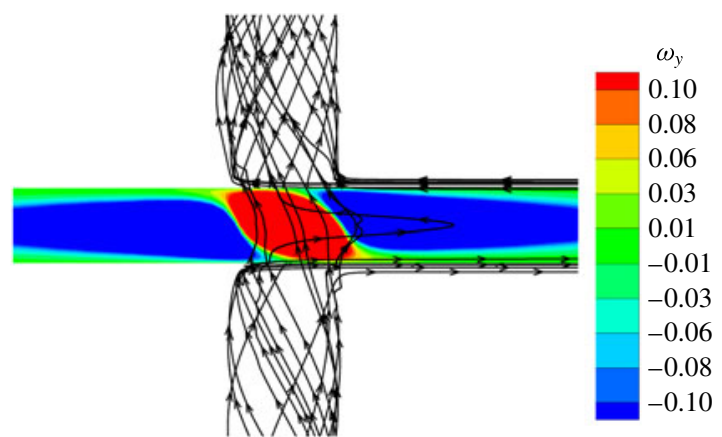

FIGURE 9. Contour plots from Case 13 of $\omega_{y}(a)$ in the $x-y$ plane and $(b)$ on the blade surface projected onto the $y-z$ plane, viewed from a perspective looking in the $x$-coordinate direction, at $t=6$. Vortex lines in both the vortex core and blade boundary layer are plotted in black.

\subsection{Lift force on the blade}

As a blade penetrates into the core of a vortex with axial flow, a transient lift force forms which peaks and then diminishes during the cutting period. As discussed in $\S 3$, a steady-state lift force can also occur due to the difference in vortex core radius across the blade surface after cutting of the vortex is complete. In this section, the effect of the different dimensionless parameters on the lift force is examined using full Navier-Stokes simulations for the different cases considered in the study. A plot showing the typical variation of the lift coefficient $C_{L}$ with time is given in figure 10 . The lift force on the blade increases as the blade penetrates into the vortex core, peaks when the blade leading edge is approximately two-thirds of the way through the cutting process, and then decreases with further time as the uncut portion of the vortex core is stretched over the blade leading edge. The three stages of vortex cutting identified by Saunders \& Marshall (2015) are indicated using vertical lines in figure 10 .

The primary cause of the large transient lift force is the unequal pressure distribution on the top and bottom blade surfaces. A time series showing pressure contours in the $x-y$ plane is given in figure 11, where the leading and trailing edge of the vortex core are marked with dashed lines. Prior to penetration of the blade leading edge into the vortex core (figure 11a), the pressure distribution is approximately symmetric over the blade centreline surface $y=0$. As the blade penetrates into the vortex core (figure 11b), the upward axial flow increases the pressure on the lower surface of the blade and decreases the pressure on the upper surface, leading to development of an asymmetric pressure profile. As cutting ends and the uncut core is stretched over the blade leading edge (figure 11c), the pressure again approaches a nearly symmetric condition and the net force on the blade decreases.

The effect of the vortex axial flow on the shear and pressure distributions on the blade surface is shown in figures 12 and 13. Figure 12(a,b) shows the change in shear stress on the blade surface between the time at which the peak cutting force occurs and that acting on a blade immersed in a uniform free stream flow with no vortex. In figure 12(a), the change in shear stress is plotted as a function of the spanwise distance $z$ along the blade leading edge $(x=y=0)$. The largest shear stress occurs in the centre of the span, where the vortex core impacts the blade. Figure $12(b)$ shows 


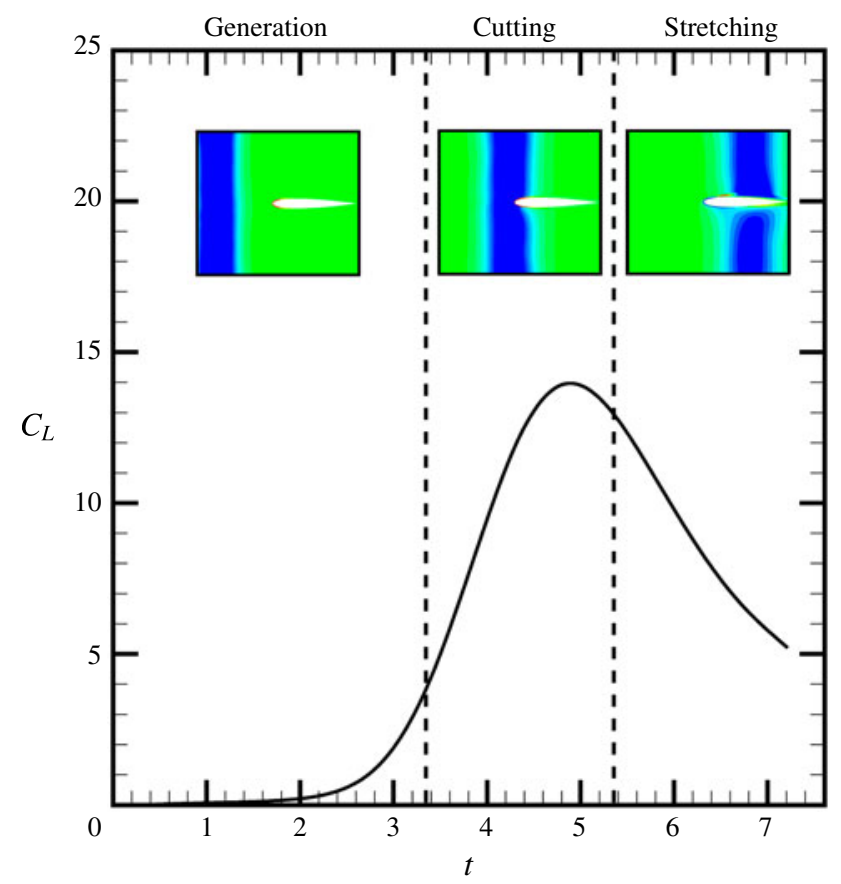

FIgURE 10. Time variation of the lift coefficient for Case 5. The three phases of vortex cutting are identified on the plot using vertical dashed lines.

(a)

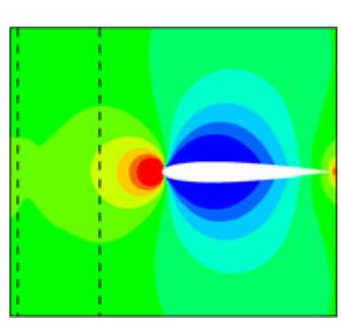

(b)

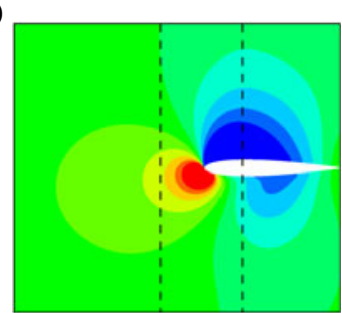

(c)

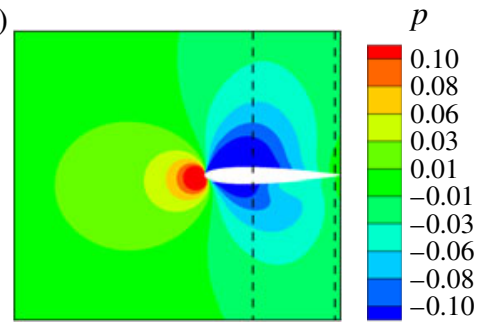

FigurE 11. Time series of pressure contours in the $x-y$ plane for Case 5 at $(a) t=1.8$, (b) 4.2 and (c) 6.6. Edges of the vortex core are indicated with dashed lines.

the change in shear stress as a function of arc length $\xi$ along a curve wrapped around the blade leading edge within the $z=0$ plane. A sketching illustrating this curve is given in figure $12(e)$, where $\xi=0$ corresponds to the blade leading edge. The largest change in shear stress occurs at the leading edge of the blade. The asymmetrical change in shear stress along the top and bottom surfaces of the blade front is due to the axial flow within the vortex core, where the axial flow combines with the free stream velocity to increase the shear stress on the blade upper surface and to decrease the shear stress on the blade lower surface. In figure 12(c), the maximum spanwise shear stress on the blade leading edge is plotted versus dimensionless time. The peak in spanwise shear stress occurs approximately two-thirds of the way through the vortex cutting process, corresponding to the peak in lift coefficient in figure 10 . In figure $12(d)$, the maximum axial velocity in the blade centre plane $y=0$ is plotted versus dimensionless time. Again, the peak occurs at approximately two-thirds of the 

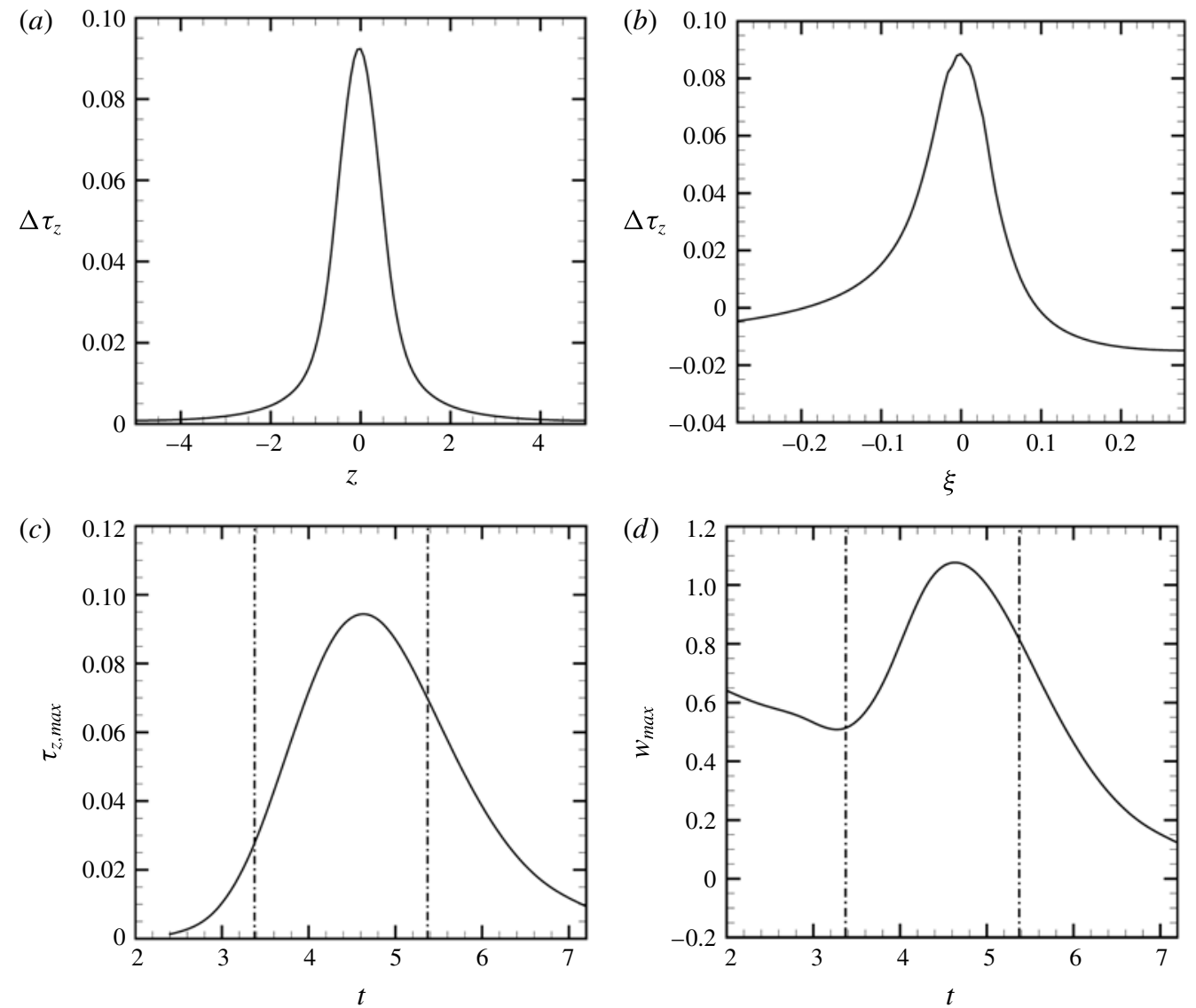

(e)

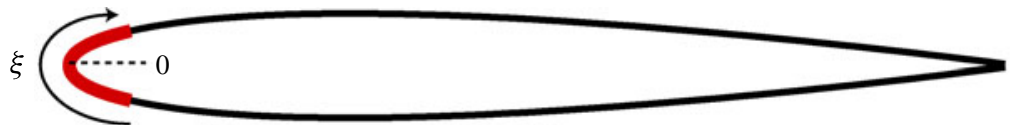

FIGURE 12. (Colour online) Difference in the spanwise shear stress $\Delta \tau_{z}$ between computations with and without the vortex present $(a)$ along the blade leading edge as a function of spanwise length $z$ and $(b)$ along the blade surface as a function of arc length $\xi$ in the $z=0$ plane (see $e$ ) at time $t=4.8$. Also shown are the maximum values of $(c)$ spanwise shear stress $\tau_{z}$ along the blade leading edge and $(d)$ axial velocity $w$ within the vortex core on the plane $y=0$ as functions of the dimensionless time. In $(c)$ and $(d)$, the nominal vortex cutting starting and ending times are indicated by vertical dashed-dotted lines. The simulations are for Case 5.

way through the vortex cutting process. As the cutting process begins, the maximum value of axial velocity increases due to the deformation of the vortex core as it is cut by the blade. After cutting, the maximum value of axial velocity in the blade centre plane decreases as the vortex compression and expansion waves propagate away from the blade, leaving regions of the vortex with low axial flow near the blade.

Figure 13 shows how the pressure distribution on the blade surface is changed by penetration of the blade into a vortex with axial flow. Figure 13(a,b) shows the change in pressure on the blade surface between the time at which the peak cutting 
(a)

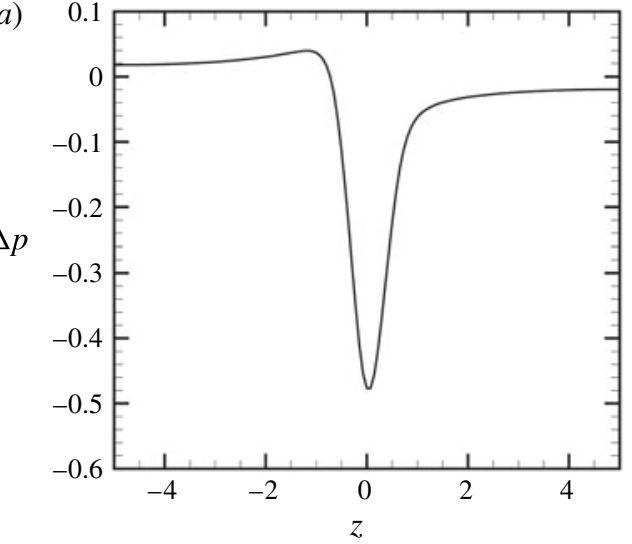

(c)

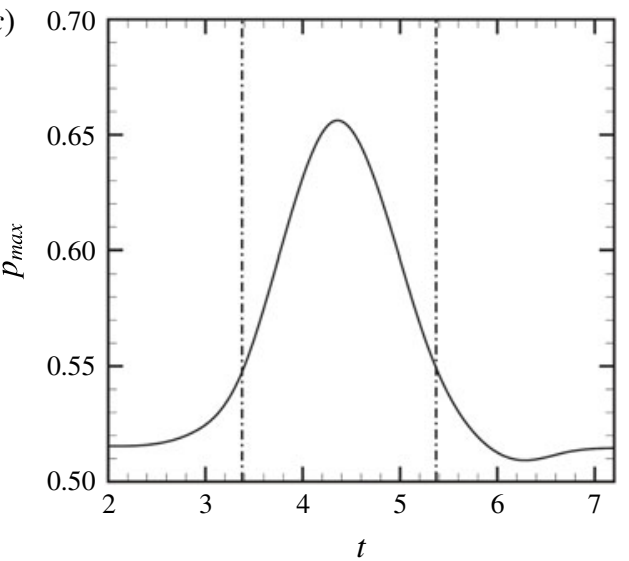

(b)

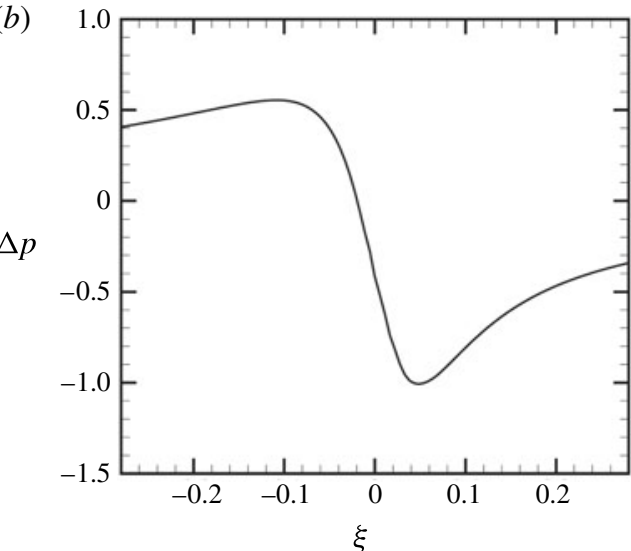

(d)

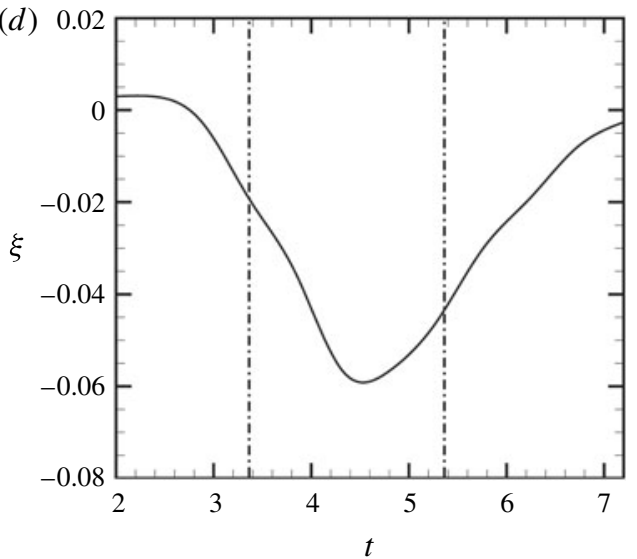

FIGURE 13. Difference in surface pressure $\Delta p$ between computations with and without the vortex present $(a)$ along the blade leading edge as a function of spanwise length $z$ and $(b)$ along the blade surface as a function of arc length $\xi$ in the $z=0$ plane at time $t=4.8$. Also shown are the maximum values of $(c)$ pressure within the plane $z=0$ and (d) value of $\xi$ corresponding to the maximum surface pressure position as functions of dimensionless time. In $(c)$ and $(d)$, the nominal vortex cutting starting and ending times are indicated by vertical dashed-dotted lines. The simulations are for Case 5.

force occurs and that acting on a blade immersed in a uniform free stream flow with no vortex. The change in pressure is plotted versus the spanwise distance $z$ in figure 13(a), showing a sharp decrease in pressure in the centre region where the vortex impinges on the blade leading edge. The pressure change is asymmetric, however, with a slightly increased pressure on the left of the vortex $(z<0)$ and a slightly decreased pressure on the right of the vortex $(z>0)$. This asymmetry is a result of the direction of the vortex swirl relative to the ambient uniform flow. The pressure change is plotted in figure $13(b)$ as a function of arc length $\xi$ on the curve wrapping about the blade front shown in figure 12(e). The pressure increases on the bottom surface of the blade and decreases on the top surface, both of which occur due to interaction of the blade with the vortex axial flow. The maximum pressure on the blade over the $z=0$ plane centre span is plotted versus dimensionless time in figure $13(c)$, and the value of arc length $\xi$ at the location of maximum pressure is 
plotted in figure $13(d)$. The pressure maximum occurs half-way through the cutting process, when the centre of the vortex core is located at the blade leading edge. As seen in figure $13(d)$, the pressure maximum is initially located at the blade leading edge, but as the blade penetrates into the vortex core the location of maximum pressure shifts downward (such that $\xi<0$ ). Half-way through the cutting process the location of the pressure maximum begins to return toward the blade leading edge (figure 13d).

The maximum lift coefficient for the different cases examined in table 1 was calculated and the results were compared with those predicted by the plug-flow model $(\S 4)$ and the scaling analysis for the transient force $(\S 2)$. In conducting this comparison, we note that the vortex structure is different in the two problems. The models used for the plug-flow computations were based on an assumption of uniform vorticity across the core, or a Rankine vortex. On the other hand, the dissipative character of the full Navier-Stokes calculations makes a Gaussian vortex a much better choice for this study. Because of these differences in vortex structure, as well as because of the numerous approximations incorporated into the plug-flow model, we expect only qualitative agreement in the lift coefficient trends obtained by the two approaches.

The predictions for the maximum lift coefficient from the Navier-Stokes simulations listed in table 1 are plotted as a function of the three dimensionless parameters $A, I$ and $T$ in figure $14(a-c)$. The data from Cases 1-6 are shown on a log-log plot in figure $14(a)$ as a function of the axial flow parameter $A$, with $I$ and $T$ held constant. Over the interval in $A$ evaluated $(0.06 \leqslant A \leqslant 11)$, the maximum lift coefficient predictions all fall on a straight line with slope -1 , indicating inverse dependence of $C_{L, \max }$ on $A$ over this range of values. The levelling out of the lift force observed for the steady-state force in figure $4(a)$ for $A>1$ is not evident in figure 14(a), which suggests that the maximum lift for these simulations is dominated by the transient lift force.

The influence of impact parameter on the lift coefficient was evaluated by plotting $C_{L, \max }$ as a function of $I$ in figure $14(b)$ for Cases 3 and 7-10, with $A$ and $T$ held fixed. The predicted lift coefficient values are observed to vary approximately linearly with a slope close to unity as $I$ varies over the interval $1.4 \leqslant I \leqslant 45$, as indicated by the solid line in figure $14(b)$. The increase in $C_{L, \text { max }}$ value above this line for the case with low impact parameter $(I=1.4)$ and the decrease in $C_{L, \max }$ value below this line for the case with high impact parameter $(I=44.5)$ are consistent with similar trends for the plug-flow model evident in figure $4(b)$. The flattening out of the lift force prediction for $I=1.4$ is similar to the effect of the steady-state lift force as observed in figure $4(b)$, whereas the transient lift force seems to dominate for the other cases plotted in figure $14(b)$. We also note that cases with small values of the impact parameter can exhibit shedding of vorticity from the blade boundary layer prior to impact of the vortex onto the blade surface (Krishnamoorthy \& Marshall 1998), which imposes a lower limit of approximately unity on the value of $I$, below which the vortex-blade interaction problem is fundamentally altered.

The influence of thickness parameter on the lift coefficient was examined by plotting $C_{L, \max }$ as a function of $T$ in figure 14(c) for Cases 3 and 11-13, with $A$ and $I$ held fixed. The maximum value of lift coefficient is found to be approximately independent of the thickness parameter in the range $T \leqslant 2$ examined in the full Navier-Stokes simulations. By comparison, the plug-flow predictions in figure $4(c)$ are also nearly constant as blade thickness is varied, although they exhibit a slight increase with thickness parameter $T$ which is not evident in the full Navier-Stokes simulation results. 

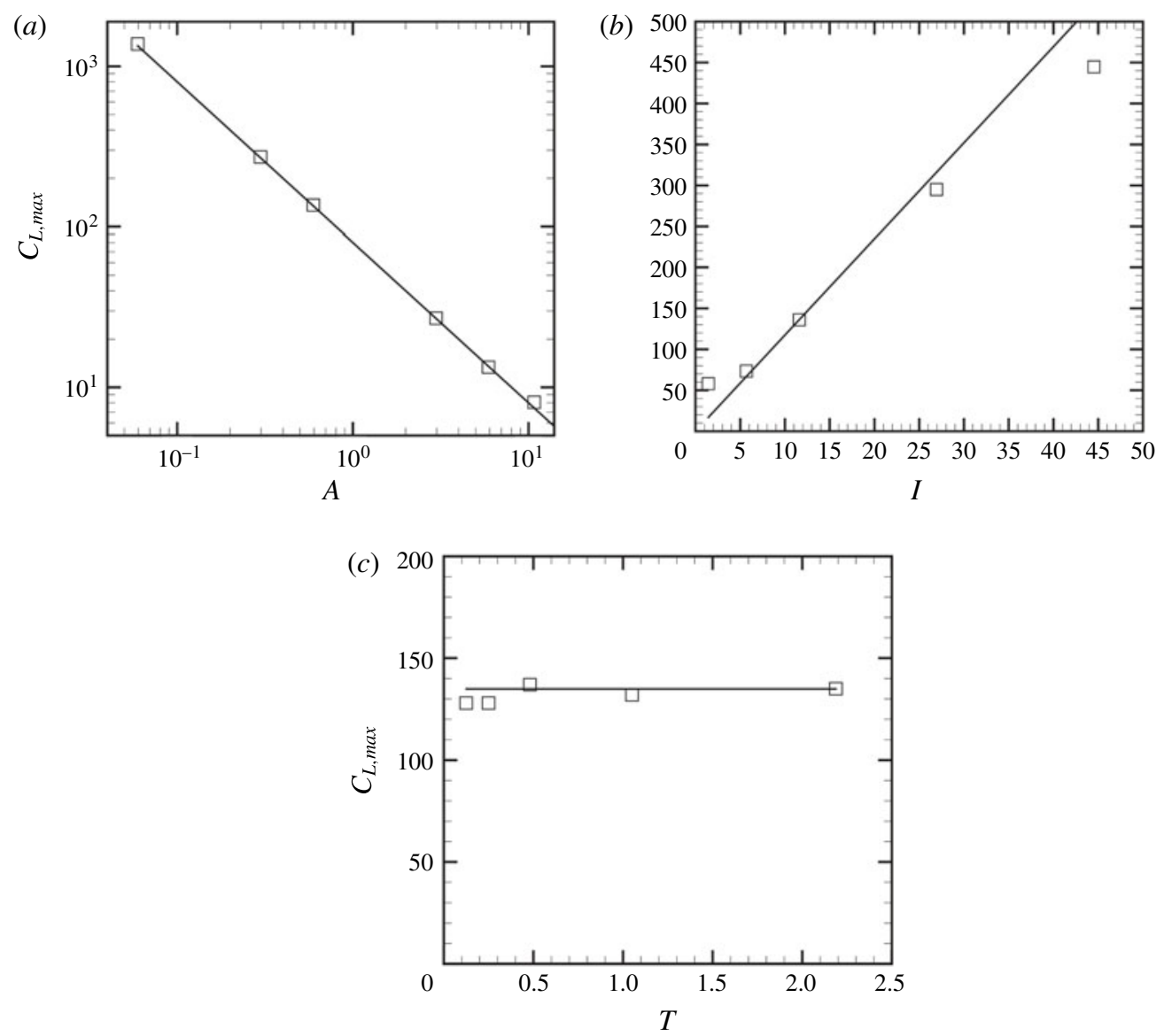

FIGURE 14. Variation of the maximum value of the lift coefficient with $(a)$ axial flow parameter $(I=11.6, T=0.48),(b)$ impact parameter $(A=0.6, T=0.48)$ and $(c)$ thickness parameter $(A=0.6, I=11.6)$. Symbols are results of full Navier-Stokes computations and lines coincide with the prediction of the scaling result (5.4).

Most of the data trends observed for the full Navier-Stokes simulation results reported in this paper indicate that the transient lift force is the dominant force controlling the maximum value of lift. The scaling analysis for the transient lift force in $\S 2$ yields the estimate (2.5) for the maximum value of the lift coefficient. In keeping with the observation that the lift coefficient is nearly independent of the thickness parameter within the range of values examined in the full Navier-Stokes simulations, we find that a good fit to the computed lift coefficient data is obtained by setting $\pi\left(C_{1} T+C_{2}\right)$ in (2.5) equal to a constant value, which is obtained as approximately 7.0 by a fit to the data in figure 14, thus giving an expression for the maximum lift coefficient over the range of parameters considered in the computations as

$$
C_{L, t r, \max } \cong 7.0 I / A \text {. }
$$

The prediction from (5.4) is indicated by the solid lines in figure 14(a-c). The good fit between the scaling estimate (5.4) and our data for maximum lift coefficient suggests 


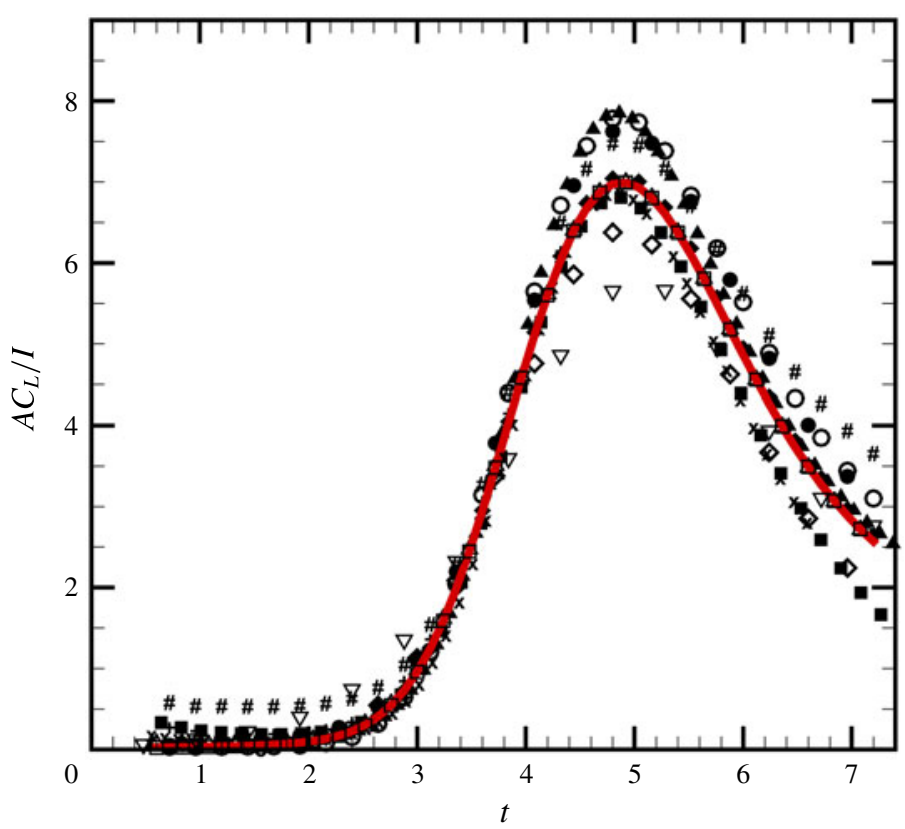

FIGURE 15. Plot showing collapse of the lift coefficient as a function of dimensionless time, with symbols indicating data from the various cases indicated in table 1 . The red curve has a maximum value of 7.0, which coincides with the scaling estimate (5.4).

that a collapse of the lift coefficient predictions for the full Navier-Stokes simulations can be obtained by plotting the ratio $A C_{L} / I$ versus dimensionless time, which is shown in figure 15. The symbols in this figure include all runs except for Case 7, which has a very low impact parameter $(I=1.4)$ and for which case the maximum lift coefficient in figure $14(b)$ is significantly above that given by (5.4).

\section{Conclusions}

The problem of blade penetration into a vortex core is important in many different problems in which vortices interact with lifting surfaces. Vortex-blade interaction causes unsteady forces on the chopping blade, which in applications such as wind energy, hydraulic pumps, helicopter aerodynamics and submarine dynamics can lead to undesirable structural vibrations or acoustic emissions. While an understanding of the various physical processes involved in blade penetration into a vortex core has slowly developed over the past two decades, there is still a great deal of uncertainty about the prediction of the vortex-blade interaction force. This is true even if we focus on a particular type of vortex-blade interaction, namely orthogonal interaction in the presence of an ambient vortex axial flow, which is a primary cause of large unsteady structural loading in these flow fields. For instance, it was noted by Marshall (1994) that the cutting of a vortex with axial flow results in a difference in core radius over the vortex core, which results in a difference in the net pressure force over the blade surface. A simple analytical model for the resulting vortex cutting force was developed using a model assuming instantaneous cutting of the vortex (which we refer to here as the steady-state vortex cutting force), but this model was not validated against full simulations or experimental results. In a later paper, Liu \& Marshall 
(2004) observed that the lift force on the blade appeared instead to be dominated by a transient force that peaks during the initial penetration of the blade leading edge into the vortex core and then decreases as the vortex is cut. They validated the computed cutting force with experiments and observed empirically that it involved an unusual scaling, which seemed to be proportional to the product of the vortex axial velocity $w_{0}$ and the free stream velocity $U$, and independent of the vortex circulation.

The current paper investigated the physics of this transient force and its relationship with the previously proposed steady-state force. This investigation was conducted using a variety of approaches - a scaling analysis of the transient penetration force, a review of the previously proposed steady-state force, a heuristic model for the vortex response to cutting based on the plug-flow approximation for the axial flow in the vortex core (similar to Saffman's (1990) model for vortex reconnection) and full Navier-Stokes simulations of the vortex-blade interaction. Each of these approaches lead to interesting insights. The scaling analysis of the transient vortex cutting force explained the empirical observation of Liu \& Marshall (2004) for why the blade lift varies as the product of $w_{0}$ and $U$, and is independent of $\Gamma$. When non-dimensionalized in terms of a lift coefficient $C_{L}$, the scaling analysis leads to the conclusion that the maximum value of $C_{L}$ for the transient force must vary in proportion to the ratio $I / A$. The steady-state vortex cutting force is just a function of the axial flow parameter $A$. The implicit expression for the steady-state force was shown to admit two asymptotic solutions (for high and low values of axial flow parameter $A$ ), which when patched together give a good explicit expression for the force over the entire range of $A$. The effect of blade penetration on the vortex in the plug-flow model (Lundgren \& Ashurst 1989) was introduced by addition of a viscous shear force on the blade, which triggered the expected response of the core radius and vortex axial flow to cutting. While the plug-flow model is very simple, it was shown to yield solutions for the blade lift coefficient that match the predictions of the transient scaling theory and the steady-state force theory in different regimes.

The full Navier-Stokes simulations were used to explore the variation of the blade surface pressure and shear stress during vortex cutting, with particular focus on the effect of the vortex axial flow on the cutting process. The predictions for lift coefficient from the full simulations were found to agree with the scaling from the transient force scaling analysis, resulting in a data collapse which yields an approximate expression for the maximum value of the blade lift coefficient during penetration into a vortex core within a region of parameter space indicated by $0.06<A<11,2 \leqslant I \leqslant 45$ and $0<T<2$. It is possible that the data collapse may also apply outside of this parameter range; however, one should be aware that in certain limits of parameter space the physical processes involved in the vortex-blade interaction change, which will likely restrict the range of applicability of this expression. For instance, for low values of impact parameter $(I<2)$, the flow is modified by shedding of vorticity from the blade leading edge prior to vortex impact. The shed vorticity wraps around the vortex core and modifies the vortex prior to impact onto the blade. For high values of thickness parameter $(T>2)$, the vortex can exhibit significant bending due to the inviscid interaction with the blade prior to impact. Vortex bending delays the impact time, stretches the vortex, and can enhance generation and shedding of boundary layer vorticity from the blade surface. For cases with sufficiently high values of the axial flow parameter, the vortex can become unstable due to the axial flow (Lessen, Singh \& Paillet 1974; Khorrami 1991; Mayer \& Powell 1992), causing both generation of bending waves and small-scale motion surrounding the vortex core. 


\section{Acknowledgement}

We gratefully acknowledge support of this work from the National Science Foundation under project DGE-1144388.

\section{REFERENCES}

Ahmadi, A. R. 1986 An experimental investigation of blade-vortex interaction at normal incidence. AIAA J. Aircraft 23 (1), 47-55.

BINDER, A. 1985 Turbulence production due to secondary vortex cutting in a turbine rotor. Trans. ASME J. Engng Gas Turbines Power 107, 1039-1046.

CARY, C. M. 1987 An experimental investigation of the chopping of helicopter main rotor tip vortices by the tail rotor. NASA CR 177457.

Churchfield, M. J., Lee, S., Michalakes, J. \& Moriarty, P. J. 2012 A numerical study of the effects of atmospheric and wake turbulence on wind turbine dynamics. J. Turbul. 13, N14.

Coton, F. N., Marshall, J. S., McD Galbraith, R. A. \& Green, R. B. 2004 Helicopter tail rotor orthogonal blade-vortex interaction. Prog. Aerosp. Sci. 40 (7), 453-486.

Doolan, C., Coton, F. \& Galbraith, R. 1999 Three-dimensional vortex interactions with a stationary blade. Aeronaut. J. 103 (1030), 578-587.

Doolan, C. J., Coton, F. N. \& Galbraith, R. A. 2001 Surface pressure measurements of the orthogonal vortex interaction. AIAA J. 38 (1), 88-95.

EARly, J., Green, R. \& Coton, F. 2002 Flow visualization of the orthogonal blade-vortex interaction using particle image velocimetry. Aeronaut. J. 106 (1057), 137-145.

Felli, M., CAmussi, R.\& GuJ, G. 2009 Experimental analysis of the flow field around a propellerrudder configuration. Exp. Fluids 46, 147-164.

Felli, M. \& FAlchi, M. 2011 Propeller tip and hub vortex dynamics in the interaction with a rudder. Exp. Fluids 51, 1385-1402.

Filippone, A. \& AfGAN, I. 2008 Orthogonal blade-vortex interaction on a helicopter tail rotor. AIAA J. 46 (6), 1476-1489.

Green, R. B., Coton, F. N. \& Early, J. M. 2006 On the three-dimensional nature of the orthogonal blade-vortex interaction. Exp. Fluids 41, 749-761.

Green, R., Doolan, C. \& CANnON, R. 2000 Measurements of the orthogonal blade-vortex interaction using a particle image velocimetry technique. Exp. Fluids 29, 369-379.

Howe, M. S. 1989 On unsteady surface forces, and sound produced by the normal chopping of a rectilinear vortex. J. Fluid Mech. 206, 131-153.

ISSA, R. 1985 Solution of the implicit discretized fluid flow equations by operator splitting. J. Comput. Phys. 62, 40-65.

Johnston, R. T. \& Sullivan, J. P. 1992 Unsteady wing surface pressures in the wake of a propeller. AIAA Paper 92-0277.

Khorrami, M. R. 1991 On the viscous modes of instability of a trailing line vortex. J. Fluid Mech. 225, 197-212.

Krishnamoorthy, S. \& MARShall, J. S. 1994 An experimental investigation of 'vortex shocks'. Phys. Fluids 6 (11), 3737-3741.

Krishnamoorthy, S. \& MARShall, J. S. 1998 Three-dimensional blade-vortex interaction in the strong-vortex regime. Phys. Fluids 10 (11), 2828-2845.

LAI, Y. G. 2000 Unstructured grid arbitrarily shaped element method for fluid flow simulation. AIAA J. 38 (12), 2246-2252.

Lee, J., Burggraf, O. \& Conlisk, A. 1998 On the impulsive blocking of a vortex jet. J. Fluid Mech. 369, 301-331.

Lessen, M., Singh, P. J. \& Paillet, F. 1974 The instability of a trailing line vortex. Part 1. Inviscid theory. J. Fluid Mech. 63, 753-763.

Leverton, J. W., Pollard, J. S. \& Wills, C. R. 1977 Main rotor wake/tail rotor interaction. Vertica 1, 213-221. 
LiU, X. \& Marshall, J. S. 2004 Blade penetration into a vortex core with and without axial core flow. J. Fluid Mech. 519, 81-103.

Lundgren, T. S. \& Ashurst, W. T. 1989 Area-varying waves on curved vortex tubes with application to vortex breakdown. J. Fluid Mech. 200, 283-307.

Marshall, J. S. 1991 A general theory of curved vortices with circular cross-section and variable core area. J. Fluid Mech. 229, 311-338.

Marshall, J. S. 1994 Vortex cutting by a blade. Part I. General theory and a simple solution. AIAA J. 32 (6), 1145-1150.

Marshall, J. S. \& GRANT, J. R. 1996 Penetration of a blade into a vortex core: vorticity response and unsteady blade forces. J. Fluid Mech. 306, 83-109.

Marshall, J. S. \& Krishnamoorthy, S. 1997 On the instantaneous cutting of a columnar vortex with non-zero axial flow. J. Fluid Mech. 351, 41-74.

Marshall, J. S. \& Yalamanchili, R. 1994 Vortex cutting by a blade. Part II. Computations of vortex response. AIAA J. 32 (7), 1428-1436.

MAYer, E. W. \& Powell, K. G. 1992 Viscous and inviscid instabilities of a trailing vortex. J. Fluid Mech. 245, 91-114.

Nagahara, T., Sato, T. \& OKamura, T. 2001 Effect of the submerged vortex cavitation occurred in pump suction intake on hydraulic forces of mixed flow pump impeller. In CAV 2001: 4th International Symposium on Cavitation, June 20-23, California Institute of Technology, Pasadena, CA, USA.

PAterson, R. W. \& AMiet, R. K. 1979 Noise of a model helicopter rotor due to ingestion of turbulence. NASA Tech. Rep. NASA CR 2313.

Saffman, P. G. 1990 A model of vortex reconnection. J. Fluid Mech. 212, 395-402.

SAUnders, D. C. \& MARshall, J. S. 2015 Vorticity reconnection during vortex cutting by a blade. J. Fluid Mech. 782, 37-62.

Shafit, S., Obermaier, H., Linn, R., Koo, E., Hlawitschka, M., Garth, C., Hamann, B. \& JOY, K. I. 2013 Visualization and analysis of vortex-turbine intersections in wind farms. IEEE Trans. Vis. Comput. Graphics 19 (9), 1579-1591.

Shelley, M. J., Meiron, D. I. \& OrszaG, S. A. 1993 Dynamical aspects of vortex reconnection of perturbed anti-parallel vortex tubes. J. Fluid Mech. 246, 613-652.

SheridAN, P. F. \& SMITH, R. P. 1980 Interactional aerodynamics - a new challenge to helicopter technology. J. Am. Helicopter Soc. 25 (1), 3-21.

Wang, T., Doolan, C. J., Coton, F. N.\& Galbraith, R. A. M. 2002 Experimental study of the three-dimensionality of orthogonal blade-vortex interaction. AIAA J. 40 (10), 2037-2046.

Weigand, A. 1993 The response of a vortex ring to a transient spatial cut. $\mathrm{PhD}$ thesis, University of California, San Diego.

YILDIRIM, E. \& HILliER, R. 2013 Numerical modeling of the impulsive orthogonal cutting of a trailing vortex. Shock Waves 23, 369-379. 